\title{
Generalized universal covering spaces and the shape group
}

\author{
by
}

\section{Hanspeter Fischer (Muncie, IN) and Andreas Zastrow (Gdańsk)}

\begin{abstract}
If a paracompact Hausdorff space $X$ admits a (classical) universal covering space, then the natural homomorphism $\varphi: \pi_{1}(X) \rightarrow \check{\pi}_{1}(X)$ from the fundamental group to its first shape homotopy group is an isomorphism. We present a partial converse to this result: a path-connected topological space $X$ admits a generalized universal covering space if $\varphi: \pi_{1}(X) \rightarrow \check{\pi}_{1}(X)$ is injective.

This generalized notion of universal covering $p: \widetilde{X} \rightarrow X$ enjoys most of the usual properties, with the possible exception of evenly covered neighborhoods: the space $\widetilde{X}$ is path-connected, locally path-connected and simply-connected and the continuous surjection $p: \widetilde{X} \rightarrow X$ is universally characterized by the usual general lifting properties. (If $X$ is first countable, then $p: \widetilde{X} \rightarrow X$ is already characterized by the unique lifting of paths and their homotopies.) In particular, the group of covering transformations $G=\operatorname{Aut}(\widetilde{X} \stackrel{p}{\rightarrow} X)$ is isomorphic to $\pi_{1}(X)$ and it acts freely and transitively on every fiber. If $X$ is locally path-connected, then the quotient $\widetilde{X} / G$ is homeomorphic to $X$. If $X$ is Hausdorff or metrizable, then so is $\tilde{X}$, and in the latter case $G$ can be made to act by isometry. If $X$ is path-connected, locally path-connected and semilocally simply-connected, then $p: \widetilde{X} \rightarrow X$ agrees with the classical universal covering.

A necessary condition for the standard construction to yield a generalized universal covering is that $X$ be homotopically Hausdorff, which is also sufficient if $\pi_{1}(X)$ is countable. Spaces $X$ for which $\varphi: \pi_{1}(X) \rightarrow \check{\pi}_{1}(X)$ is known to be injective include all subsets of closed surfaces, all 1-dimensional separable metric spaces (which we prove to be covered by topological $\mathbb{R}$-trees), as well as so-called trees of manifolds which arise, for example, as boundaries of certain Coxeter groups.

We also obtain generalized regular coverings, relative to some special normal subgroups of $\pi_{1}(X)$, and provide the appropriate relative version of being homotopically Hausdorff, along with its corresponding properties.
\end{abstract}

General Assumption. Throughout this article, we consider a pathconnected topological space $X$ with base point $x_{0} \in X$.

2000 Mathematics Subject Classification: Primary 55R65; Secondary 57M10, 55Q07.

Key words and phrases: generalized universal covering, first shape homotopy group, generalized regular covering.

The authors gratefully acknowledge the support of this research by the Faculty Internal Grants Program of Ball State University, the University of Gdańsk (BW UG 5100-50096-5) and the Polish Ministry of Science (KBN 0524/H03/2006/31). 
1. Introduction. Recall that a continuous map $p: \bar{X} \rightarrow X$ is called a covering of $X$, and $\bar{X}$ is called a covering space of $X$, if for every $x \in X$ there is an open subset $U$ of $X$ with $x \in U$ and such that $U$ is evenly covered by $p$, that is, $p^{-1}(U)$ is the disjoint union of open subsets of $\bar{X}$ each of which is mapped homeomorphically onto $U$ by $p$.

In the classical theory, one assumes that $X$ is, in addition, locally pathconnected and wishes to classify all path-connected covering spaces of $X$ and to find among them a universal covering space, that is, a covering $p: \widetilde{X} \rightarrow X$ with the property that for every covering $q: \bar{X} \rightarrow X$ by a path-connected space $\bar{X}$ there is a covering $\bar{q}: \widetilde{X} \rightarrow \bar{X}$ such that $q \circ \bar{q}=p$. If $X$ is locally pathconnected, we have the following well-known result, which can be found, for example, in [22] and [25]:

Every simply-connected covering space of $X$ is a universal covering space. Moreover, $X$ admits a simply-connected covering space if and only if $X$ is semilocally simply-connected, in which case the coverings $p:(\bar{X}, \bar{x}) \rightarrow$ $\left(X, x_{0}\right)$ with path-connected $\bar{X}$ are in direct correspondence with the conjugacy classes of subgroups of $\pi_{1}\left(X, x_{0}\right)$, via the monomorphism $p_{\#}: \pi_{1}(\bar{X}, \bar{x})$ $\rightarrow \pi_{1}\left(X, x_{0}\right)$.

Outside of semilocally simply-connected spaces, the theory is not as pleasant. This is unfortunate, because non-semilocally simply-connected spaces, such as the Pontryagin surface $\Pi_{2}$, the 1-dimensional Menger universal curve, the Hawaiian Earring and other locally complicated planar sets are routinely studied in various areas of topology, fractal geometry and dynamical systems. Accordingly, one would like to generalize the notion of universal covering space so as to include such spaces.

While it is possible, based on Fox's concept of overlay, to classify specific types of (classical) covering spaces of non-semilocally simply-connected spaces via the fundamental pro-group pro- $\pi_{1}\left(X, x_{0}\right)[12-15,19,22]$, no universal covering space might be available.

An improved correspondence result was obtained in [19] by relaxing the definitions of "space" and "fundamental group". However, since the generalized notion of "covering space" in that paper is still modeled on a local isomorphism of structures, not every planar, path-connected and locally path-connected, topological space ends up having a "simply-connected" covering space [19, Ex. 9, §11].

Every attempt to generalize the notion of universal covering space is invariably tied to a subjective list of those properties which are deemed indispensable, and another list of properties, which must necessarily be discarded. Of course, the proper balance between these two lists will always depend on the task at hand. It seems, however, that for most applications the particular usefulness of a simply-connected covering space does not lie 
in the evenly covered neighborhoods, but rather in the following properties:

$\left(\mathrm{U}_{1}\right)$ The space $\widetilde{X}$ is path-connected, locally path-connected and simplyconnected.

$\left(\mathrm{U}_{2}\right)$ The map $p: \widetilde{X} \rightarrow X$ is a continuous surjection.

$\left(\mathrm{U}_{3}\right)$ For every path-connected and locally path-connected topological space $Y$, for every continuous function $f:(Y, y) \rightarrow(X, x)$ with $f_{\#}\left(\pi_{1}(Y, y)\right)=1$, and for every $\widetilde{x}$ in $\widetilde{X}$ with $p(\widetilde{x})=x$, there exists a unique continuous lift $g:(Y, y) \rightarrow(\widetilde{X}, \widetilde{x})$ with $p \circ g=f$.

$\left(\mathrm{U}_{4}\right)$ The group of covering transformations $\operatorname{Aut}(\widetilde{X} \stackrel{p}{\rightarrow} X)$ is isomorphic to $\pi_{1}\left(X, x_{0}\right)$ and it acts freely and transitively on every fiber $p^{-1}(\{x\})$ with $x \in X$.

$\left(\mathrm{U}_{5}\right)$ The map $p: \widetilde{X} \rightarrow X$ is open so that $\tilde{X} / G \approx X$, where $G=$ $\operatorname{Aut}(\widetilde{X} \stackrel{p}{\rightarrow} X)$.

In the absence of a classical universal covering, still certain Hurewicz fibrations [25] and certain Serre fibrations [2], [10, §5] $p: E \rightarrow X$ are sometimes available, with simply-connected $E$ and additional helpful properties. However, these fibrations lack, in general, most of the properties on the above list, notably the local path-connectivity of $E$, on which the other properties hinge - even if $X$ is locally path-connected.

The approach of this paper is fundamentally different from all of the above. Seeking the middle ground between letting go of local homeomorphisms and considering very general fibrations, we examine the standard construction of the classical universal covering and ask the question: under what circumstances will it have Properties $\mathrm{U}_{1}-\mathrm{U}_{5}$ ? This approach is in the spirit of [28] as well as [3].

1.1. On Properties $\mathrm{U}_{1}-\mathrm{U}_{5}$. Properties $\mathrm{U}_{1}, \mathrm{U}_{2}$ and $\mathrm{U}_{3}$ uniquely characterize $p: \widetilde{X} \rightarrow X$ in the usual way: if any two maps $p_{1}:\left(\widetilde{X}_{1}, \widetilde{x}_{1}\right) \rightarrow(X, x)$ and $p_{2}:\left(\widetilde{X}_{2}, \widetilde{x}_{2}\right) \rightarrow(X, x)$ satisfy Properties $\mathrm{U}_{1}-\mathrm{U}_{3}$, then there are (unique) lifts $g_{1}:\left(\widetilde{X}_{1}, \widetilde{x}_{1}\right) \rightarrow\left(\widetilde{X}_{2}, \widetilde{x}_{2}\right)$ and $g_{2}:\left(\widetilde{X}_{2}, \widetilde{x}_{2}\right) \rightarrow\left(\widetilde{X}_{1}, \widetilde{x}_{1}\right)$ with $p_{2} \circ g_{1}=p_{1}$ and $p_{1} \circ g_{2}=p_{2}$. Since both $g_{2} \circ g_{1}$ and id $\tilde{X}_{1}$ are lifts with $p_{1} \circ\left(g_{2} \circ g_{1}\right)=p_{1}$ and $p_{1} \circ \mathrm{id}_{\widetilde{X}_{1}}=p_{1}$, and since such lifts are assumed to be unique, we have $g_{2} \circ g_{1}=\mathrm{id}_{\widetilde{X}_{1}}$. Similarly, $g_{2} \circ g_{1}=\mathrm{id}_{\widetilde{X}_{2}}$. Hence $g_{1}: \widetilde{X}_{1} \rightarrow \widetilde{X}_{2}$ is a homeomorphism with $p_{2} \circ g_{1}=p_{1}$. That is, $p_{1}: \widetilde{X}_{1} \rightarrow X$ and $p_{2}: \widetilde{X}_{2} \rightarrow X$ are isomorphic.

Properties $\mathrm{U}_{1}-\mathrm{U}_{3}$ combined imply Property $\mathrm{U}_{4}$ : firstly, if we take $p_{1}=$ $p_{2}=p$ and $\widetilde{X}_{1}=\widetilde{X}_{2}=\widetilde{X}$ in the previous paragraph, we see that the group of covering transformations Aut $(\widetilde{X} \stackrel{p}{\rightarrow} X)$ (i.e. the group of homeomorphisms $g: \widetilde{X} \rightarrow \widetilde{X}$ with $p \circ g=p)$ acts freely and transitively on every fiber $p^{-1}(\{x\})$. Moreover, for a fixed $\widetilde{x}_{0} \in \widetilde{X}$ with $p\left(\widetilde{x}_{0}\right)=x_{0}$, an isomorphism 
$\Psi: \operatorname{Aut}(\tilde{X} \stackrel{p}{\rightarrow} X) \rightarrow \pi_{1}\left(X, x_{0}\right)$ is given by $\Psi(f)=[p \circ \alpha]$, where $\alpha$ is any path in $\widetilde{X}$ from $\widetilde{x}_{0}$ to $f\left(\widetilde{x}_{0}\right)$.

If $X$ is first countable, then Property $\mathrm{U}_{3}$ is already captured by the unique lifting of paths and their homotopies (see Proposition 5.1). Moreover, Properties $\mathrm{U}_{1}-\mathrm{U}_{3}$ imply Property $\mathrm{U}_{5}$, provided $X$ is locally path-connected and first countable (see Remark 5.2). In turn, we cannot expect Property $\mathrm{U}_{5}$ to hold in the presence of local path-connectivity of $\widetilde{X}$ in conjunction with Property $\mathrm{U}_{2}$, unless $X$ itself is locally path-connected. (See Remark 4.17 for the non-locally path-connected case.) However, we will never assume $X$ to be locally path-connected without explicit mention, because several results of this article apply to non-locally path-connected spaces as well.

We therefore make the following definition.

Definition. If a map $p: \widetilde{X} \rightarrow X$ satisfies Properties $\mathrm{U}_{1}, \mathrm{U}_{2}$ and $\mathrm{U}_{3}$, then we call it the generalized universal covering of $X$ and we call $\widetilde{X}$ the generalized universal covering space of $X$.

1.2. Fibrations and higher homotopy groups. Every generalized universal covering $p: \widetilde{X} \rightarrow X$ is a Serre fibration with unique path lifting. (Recall that $p: \widetilde{X} \rightarrow X$ is called a Serre fibration if it has the homotopy lifting property with respect to $Y=[0,1]^{n}$ for all $n \geq 0$. That is, for every map $F: Y \times[0,1] \rightarrow X$ and every partial lift $g: Y \times\{0\} \rightarrow \widetilde{X}$ with $p \circ g=$ $F \circ$ incl there is a map $G: Y \times[0,1] \rightarrow \widetilde{X}$ such that $G \circ$ incl $=g$ and $p \circ G=F)$. Consequently, the homomorphisms $p_{\#}: \pi_{i}(\widetilde{X}) \rightarrow \pi_{i}(X)$ are isomorphisms for $i>1$. However, a generalized universal covering need not be a covering in the classical sense or even a Hurewicz fibration: although Property $\mathrm{U}_{4}$ implies that the cardinality of every fiber $p^{-1}(\{x\})$ is that of $\pi_{1}(X)$, Example 4.15 features non-homeomorphic fibers.

1.3. Summary of results. A necessary condition for the standard construction to yield a generalized universal covering is that $X$ be homotopically Hausdorff, that is, for every $x \in X$ only the trivial element of $\pi_{1}(X, x)$ can be represented by arbitrarily small loops (Definition 2.9 and Lemma 2.11). If $\pi_{1}\left(X, x_{0}\right)$ is countable, then this property is also sufficient (Theorem 4.4). This is not surprising, for if $X$ is first countable and homotopically Hausdorff with countable $\pi_{1}\left(X, x_{0}\right)$, then $X$ is actually semilocally simply-connected (Proposition 4.6). In turn, it is well-known that every (connected) locally path-connected, semilocally simply-connected, separable metric space has a countable fundamental group (see also Theorem 4.1).

Therefore, this article is mainly concerned with finding a condition under which spaces with uncountable fundamental group admit generalized universal coverings. As we shall see, the only obstruction is the unique path lifting property (Example 2.7 and Proposition 2.14). 
We begin our investigation with the observation that if $X$ is locally pathconnected, semilocally simply-connected and paracompact Hausdorff, then the natural homomorphism $\varphi: \pi_{1}\left(X, x_{0}\right) \rightarrow \check{\pi}_{1}\left(X, x_{0}\right)$ from the fundamental group of $X$ to its first shape homotopy group is an isomorphism (Theorem 4.1). Our main result (Theorem 4.10) is a partial converse, namely that if the homomorphism $\varphi: \pi_{1}\left(X, x_{0}\right) \rightarrow \check{\pi}_{1}\left(X, x_{0}\right)$ is injective, then the standard construction of $p: \widetilde{X} \rightarrow X$ via homotopy classes of paths, when given the correct topology, yields a generalized universal covering. Moreover, $p: \widetilde{X} \rightarrow X$ is an open map whenever $X$ is locally path-connected. Should $X$ be Hausdorff or metrizable, then $\widetilde{X}$ inherits these properties, and in the latter case there is a metric for $\widetilde{X}$ such that the covering transformations act by isometry.

Spaces $X$ for which $\varphi: \pi_{1}\left(X, x_{0}\right) \rightarrow \check{\pi}_{1}\left(X, x_{0}\right)$ is known to be injective include all planar sets and, more generally, all subsets of closed surfaces, all 1-dimensional (Hausdorff) compacta and 1-dimensional separable metric spaces, the above-mentioned Pontryagin surface and, more generally, trees of manifolds which arise, for example, as boundaries of groups. (See Examples 4.13-4.16.) Our main theorem therefore equips all of these spaces with a generalized universal covering. The generalized universal covering space of a 1-dimensional (path-connected) separable metric space will turn out to be (topologically) an $\mathbb{R}$-tree. In the case of the Hawaiian Earring, we will give an explicit combinatorial description of the generalized universal covering.

We show that injectivity of $\varphi: \pi_{1}(X) \rightarrow \check{\pi}_{1}(X)$ does not guarantee the existence of all generalized intermediate coverings (Example 6.2). However, we obtain generalized regular coverings, relative to some special normal subgroups of $\pi_{1}(X)$, and provide the appropriate relative version of being homotopically Hausdorff, along with its corresponding properties. (Proposition 6.4 generalizes Lemma 2.11; Proposition 6.6 generalizes Proposition 4.6; Theorem 6.10 generalizes Theorems 4.4 and 4.10.)

1.4. On the structure of this article. In Section 2, we examine the standard construction of a universal covering $p: \widetilde{X} \rightarrow X$ when applied to a general path-connected space $X$. We determine which topology to invoke for $\widetilde{X}$ in order to ensure that it is locally path-connected. We also list some elementary properties of $p: \widetilde{X} \rightarrow X$, the proofs of which can in many cases be adapted from the standard theory (as found in [22] and [25]), since they do not rely on semilocal simple-connectivity of $X$. While much of the introductory material in Section 2 was previously observed in [28] and [3], or belongs to folklore, detailed proofs are provided as self-contained background.

Before we state and prove the main results along with our examples in Section 4, we briefly recall the definition of the first shape homotopy group in Section 3. 
In Section 5 we prove the above-mentioned properties of first countable spaces. Finally, Section 6 is devoted to generalized regular coverings and contains the relative versions of our main results.

2. The "standard" construction. Note that a generalized universal covering space $\widetilde{X}$ of $X$, if it exists, must be in one-to-one correspondence with the homotopy classes of paths in $X$ which emanate from $x_{0}$. Accordingly, there is only one way to define the set $\widetilde{X}$ : let $\mathcal{P}(X)$ be the set of all continuous maps $\alpha:[0,1] \rightarrow X$ such that $\alpha(0)=x_{0}$. On $\mathcal{P}(X)$, consider the equivalence relation given by $\alpha \sim \beta$ if and only if $\alpha(1)=\beta(1)$ and $\alpha$ is homotopic to $\beta$ within $X$, relative to their common endpoints. Let $[\alpha]$ denote the equivalence class of $\alpha$ and let $\widetilde{X}$ denote the set of all such equivalence classes.

If now $\widehat{p}: \widehat{X} \rightarrow X$ is a generalized universal covering and if $\widehat{p}\left(\widehat{x}_{0}\right)=x_{0}$, then the function which assigns to a point $\widehat{x}$ of $\widehat{X}$ the homotopy class $\widehat{p}_{\#}([\alpha])$, where $\alpha$ is any path in $\widehat{X}$ from $\widehat{x}_{0}$ to $\widehat{x}$, is a bijection from $\widehat{X}$ onto $\widetilde{X}$. Consequently, there is also no ambiguity as to what the projection function $p: \widetilde{X} \rightarrow X$ ought to be: we define $p([\alpha])=\alpha(1)$.

Next, we need to decide on the correct topology for $\widetilde{X}$. For each $[\alpha] \in \widetilde{X}$ and each open subset $U$ of $X$ containing $\alpha(1)$, let $B([\alpha], U)$ denote the set of all $[\beta] \in \widetilde{X}$ for which there exists a continuous map $\gamma:[0,1] \rightarrow U$ such that $\gamma(0)=\alpha(1), \gamma(1)=\beta(1)$ and $[\beta]=[\alpha \cdot \gamma]$; where $\alpha \cdot \gamma$ denotes the usual concatenation of the paths $\alpha$ and $\gamma$. Notice that $B([\alpha], X)=\widetilde{X}$ for all $[\alpha] \in \widetilde{X}$ and that if $[\beta] \in B([\alpha], U)$, then $B([\beta], U)=B([\alpha], U)$. Moreover, if $U \subseteq V$, then $B([\alpha], U) \subseteq B([\alpha], V)$. It follows that the collection of all such sets $B([\alpha], U)$ forms a basis for a topology on $\tilde{X}$.

In the event that $X$ is locally path-connected and semilocally simplyconnected, $\widetilde{X}$ is the classical universal covering space of $X$ and $p: \widetilde{X} \rightarrow X$ is the classical universal covering of $X$ as defined in [22] and [25]. However, as the following lemma explains, there are two "standard" constructions, only one of which leads to properties $\mathrm{U}_{1}-\mathrm{U}_{5}$. In [2] and [10, $\left.\S 5\right]$, for example, $\widetilde{X}$ is equipped with the quotient topology, which it inherits from the compactopen topology on $\mathcal{P}(X)$.

Lemma 2.1. The topology on $\widetilde{X}$ generated by the basis elements $B([\alpha], U)$ is, in general, finer than the quotient topology inherited from the compactopen topology on $\mathcal{P}(X)$. If $X$ is locally path-connected and semilocally simplyconnected, then the two topologies agree. However, the compact-open topology does not, in general, render $\widetilde{X}$ locally path-connected.

Proof. Consider $\mathcal{P}(X)$ in the compact-open topology and let $q: \mathcal{P}(X) \rightarrow$ $\widetilde{X}$ be the quotient map. Let $\widetilde{U}$ be a subset of $\widetilde{X}$ which is open in the quotient topology. Then $q^{-1}(\widetilde{U})$ is open in $\mathcal{P}(X)$. Let $[\alpha] \in \widetilde{U}$. Then $\alpha \in q^{-1}(\widetilde{U})$. 
Hence, there are compact subsets $C_{1}, \ldots, C_{n}$ of $[0,1]$ and open subsets $U_{1}, \ldots, U_{n}$ of $X$ with $\alpha \in S\left(C_{1}, U_{1}\right) \cap \cdots \cap S\left(C_{n}, U_{n}\right) \subseteq q^{-1}(\widetilde{U})$, where $S\left(C_{i}, U_{i}\right)$ is the set of all continuous maps $\beta:[0,1] \rightarrow X$ with $\beta(0)=x_{0}$ and $\beta\left(C_{i}\right) \subseteq U_{i}$. Define $V=X \cap \bigcap\left\{U_{i} \mid 1 \in C_{i}\right\}$. Then $\alpha(1) \in V$. We will show that $B([\alpha], V) \subseteq \widetilde{U}$. To this end, let $[\beta] \in B([\alpha], V)$. Then $[\beta]=[\alpha \cdot \gamma]$ for some continuous map $\gamma:[0,1] \rightarrow V$ with $\gamma(0)=\alpha(1)$ and $\gamma(1)=\beta(1)$. Choose $\delta \in(0,1)$ such that $\alpha([\delta, 1]) \subseteq V$ and $C_{i} \cap[\delta, 1]=\emptyset$ whenever $1 \notin C_{i}$. Then we may assume that $\alpha(t)=\beta(t)$ for all $t \in[0, \delta]$ and that $\beta(t) \in V$ for all $t \in[\delta, 1]$. Hence, $\beta\left(C_{i}\right)=\alpha\left(C_{i}\right) \subseteq U_{i}$ if $1 \notin C_{i}$. If $1 \in C_{i}$ and $t \in C_{i}$, then either $\beta(t)=\alpha(t) \subseteq U_{i}$ (for $t \leq \delta$ ) or $\beta(t) \in V \subseteq U_{i}$ (for $t \geq \delta$ ). Therefore, $\beta \in S\left(C_{1}, U_{1}\right) \cap \cdots \cap S\left(C_{n}, U_{n}\right)$ so that $[\beta]=q(\beta) \in q\left(S\left(C_{1}, U_{1}\right) \cap \cdots \cap S\left(C_{n}, U_{n}\right)\right) \subseteq \widetilde{U}$.

Now assume that $X$ is locally path-connected and semilocally simplyconnected. Let $[\alpha] \in \widetilde{X}$ and let $U$ be an open subset of $X$ containing $\alpha(1)$. Let $[\beta] \in B([\alpha], U)$. As above, we may assume that there is a $\delta \in(0,1)$ such that $\alpha(t)=\beta(t)$ for all $t \in[0, \delta]$ and that $\beta(t) \in U$ for all $t \in$ $[\delta, 1]$. Partition $[0, \delta]$ into $\left\{0=t_{0}<t_{1}<\cdots<t_{n}=\delta\right\}$ such that each $\alpha\left(\left[t_{j}, t_{j+1}\right]\right)$ lies in some path-connected open subset $U_{j}$ of $X$ with $\pi_{1}\left(U_{j}\right) \rightarrow$ $\pi_{1}(X)$ trivial. Put $U_{n+1}=U$. Let $V_{j}$ be the path-component of $U_{j-1} \cap U_{j}$ which contains $\alpha\left(t_{j}\right)$. Then each $V_{j}$ is open in $X$. Put $W=S\left(\left[t_{0}, t_{1}\right], U_{1}\right) \cap$ $S\left(\left\{t_{1}\right\}, V_{1}\right) \cap S\left(\left[t_{1}, t_{2}\right], U_{2}\right) \cap S\left(\left\{t_{2}\right\}, V_{2}\right) \cap S\left(\left[t_{2}, t_{3}\right], U_{3}\right) \cap \cdots \cap S\left(\left\{t_{n-1}\right\}, V_{n}\right) \cap$ $S\left(\left[t_{n-1}, t_{n}\right], U_{n}\right) \cap S\left(\left\{t_{n}\right\}, V_{n}\right) \cap S([\delta, 1], U)$. Then $[\beta] \in q(W) \subseteq B([\alpha], U)$. Moreover, $q^{-1}(q(W))$ is open in $\mathcal{P}(X)$, which can be shown by a very similar argument.

The next example shows that if $\widetilde{X}$ is given the quotient topology inherited from the compact-open topology on $\mathcal{P}(X)$, then $\widetilde{X}$ might not be locally path-connected.

EXAmple 2.2. If $X=\left\{(x, y) \in \mathbb{R}^{2} \mid x^{2}+(y-1 / n)^{2}=(1 / n)^{2}\right.$ for some $n \in \mathbb{N}\}$ is the Hawaiian Earring with base point $x_{0}=(0,0)$ and if $\widetilde{X}$ is given the quotient topology inherited from the compact-open topology on $\mathcal{P}(X)$, then $\widetilde{X}$ is not locally path-connected. Indeed, if $l_{n}$ denotes the simple closed loop of $X$ of radius $1 / n$ based at $x_{0}$, then the sequence $\widetilde{x}_{n}=\left[l_{1}\right]\left[l_{n}\right]\left[l_{1}\right]$ converges to $\widetilde{x}=\left[l_{1}\right]\left[l_{1}\right]$ in this topology, although there are no small paths connecting $\widetilde{x}_{n}$ and $\widetilde{x}$.

Notation. From here on forward, we will endow $\widetilde{X}$ with the topology generated by the basis elements $B([\alpha], U)$, with $[\alpha] \in \widetilde{X}$ and $U \subseteq X$ open, and the projection function $p:\left(\widetilde{X}, \widetilde{x}_{0}\right) \rightarrow\left(X, x_{0}\right)$ will be given by $p([\alpha])=\alpha(1)$, where $\widetilde{x}_{0}$ denotes the equivalence class containing the constant path at $x_{0}$.

Lemma 2.3. The projection $p: \widetilde{X} \rightarrow X$ is a continuous surjection; it is open if and only if $X$ is locally path-connected. 
Proof. The function $p: \widetilde{X} \rightarrow X$ is surjective, because $X$ is path-connected. In order to show that $p: \widetilde{X} \rightarrow X$ is continuous, let $[\alpha] \in \widetilde{X}$ and let $U$ be an open subset of $X$ with $p([\alpha])=\alpha(1) \in U$. Then $p(B([\alpha], U)) \subseteq U$.

Finally, observe that $p(B([\alpha], U))$ equals the path-component of $U$ which contains $\alpha(1)$. Therefore, $p: \widetilde{X} \rightarrow X$ is open if and only if $X$ is locally path-connected.

The appropriate lifts always exist:

Lemma 2.4. Suppose that $Y$ is path-connected and locally path-connected, that $f:(Y, y) \rightarrow(X, x)$ is continuous with $f_{\#}\left(\pi_{1}(Y, y)\right)=1$, and that $\widetilde{x} \in \widetilde{X}$ with $p(\widetilde{x})=x$. Then there is a continuous function $g:(Y, y) \rightarrow(\widetilde{X}, \widetilde{x})$ with $p \circ g=f$. Specifically, if we write $\widetilde{x}=[\alpha]$, we can define $g: Y \rightarrow \widetilde{X}$ as follows: for $w \in Y$, choose any path $\tau:[0,1] \rightarrow Y$ from $\tau(0)=y$ to $\tau(1)=w$, and put $g(w)=[\alpha \cdot(f \circ \tau)]$.

Definition. We will refer to the map $g:(Y, y) \rightarrow(\widetilde{X}, \widetilde{x})$ defined in Lemma 2.4 as the standard lift of $f:(Y, y) \rightarrow(X, x)$ at $\widetilde{x}$.

Proof. Notice that $g$ is well-defined, because $f_{\#}\left(\pi_{1}(Y, y)\right)=1$. To verify continuity, we let $w \in Y,[\beta] \in \widetilde{X}$, and $U$ an open set of $X$, containing $\beta(1)$, such that $g(w) \in B([\beta], U)$. Say, $g(w)=[\gamma]=[\alpha \cdot(f \circ \tau)]$ as in the definition above. Since $[\gamma] \in B([\beta], U)$, we have $B([\gamma], U)=B([\beta], U)$. Since $f(w)=f(\tau(1))=\gamma(1) \in U$, we may choose a path-connected open set $V$ of $Y$ with $w \in V$ such that $f(V) \subseteq U$. We claim that $g(V) \subseteq B([\beta], U)$. To see why, let $v \in V$ and choose a continuous map $\delta:[0,1] \rightarrow V$ with $\delta(0)=w$ and $\delta(1)=v$. If we put $\eta=\alpha \cdot(f \circ \tau) \cdot(f \circ \delta)$, then $[\eta] \in B([\gamma], U)$, so that $g(v)=[\eta] \in B([\eta], U)=B([\gamma], U)=B([\beta], U)$. The fact that $g(y)=\widetilde{x}$ and $p \circ g=f$ follows straight from the definition of $g$.

LEMma 2.5. The space $\widetilde{X}$ is path-connected and locally path-connected, even if $X$ is not locally path-connected. However, $\widetilde{X}$ might not be simplyconnected.

Proof. Since $B\left(\widetilde{x}_{0}, X\right)=\widetilde{X}$, it suffices to prove that $B([\alpha], U)$ is pathconnected whenever $[\alpha] \in \widetilde{X}$ and $U$ is an open subset of $X$ containing $\alpha(1)$. To this end, let $[\beta] \in B([\alpha], U)$. Choose a continuous map $\gamma:[0,1] \rightarrow U$ with $\gamma(0)=\alpha(1), \gamma(1)=\beta(1)$, and $[\beta]=[\alpha \cdot \gamma]$. Put $\widetilde{x}=[\alpha]$ and $x=p(\widetilde{x})=\alpha(1)$. Then the standard lift $g:([0,1], 0) \rightarrow(\widetilde{X}, \widetilde{x})$ of $\gamma:([0,1], 0) \rightarrow(X, x)$ is a continuous path from $g(0)=[\alpha]$ to $g(1)=[\alpha \cdot \gamma]=[\beta]$. Moreover, for every $t \in[0,1]$, let $\tau_{t}:[0,1] \rightarrow[0, t]$ be defined by $\tau_{t}(s)=t s$. Then $g(t)=\left[\alpha \cdot\left(\gamma \circ \tau_{t}\right)\right] \in B([\alpha], U)$.

Example 2.7 shows that $\widetilde{X}$ might not be simply-connected. 
The group of covering transformations always includes the standard transformations, which are induced by the canonical action of $\pi_{1}\left(X, x_{0}\right)$ on $\widetilde{X}$. This is true, whether or not $\widetilde{X}$ is simply-connected:

Lemma 2.6. Let $\widetilde{x}_{1}, \widetilde{x}_{2} \in \widetilde{X}$ with $p\left(\widetilde{x}_{1}\right)=p\left(\widetilde{x}_{2}\right)$. Then there is a homeomorphism $g:\left(\widetilde{X}, \widetilde{x}_{1}\right) \rightarrow\left(\widetilde{X}, \widetilde{x}_{2}\right)$ with $p \circ g=p$. Indeed, for $\widetilde{x} \in \widetilde{X}$ we may define $g(\widetilde{x})=[\beta \cdot \bar{\alpha} \cdot \gamma]$, where $\widetilde{x}_{1}=[\alpha], \widetilde{x}_{2}=[\beta], \widetilde{x}=[\gamma]$ and $\bar{\alpha}(t)=\alpha(1-t)$. In particular, if we take $\widetilde{x}_{1}=\widetilde{x}_{0}$, we see that the action of $\pi_{1}\left(X, x_{0}\right)$ on $\widetilde{X}$ given by $[\beta] \cdot[\gamma]=[\beta \cdot \gamma]$ yields an isomorphism of $\pi_{1}\left(X, x_{0}\right)$ onto a subgroup of $\operatorname{Aut}(\widetilde{X} \stackrel{p}{\rightarrow} X)$.

Proof. Continuity of $g$ follows from the formula $g(B([\gamma], U))=B(g([\gamma]), U)$ and its inverse is given by $g^{-1}([\gamma])=[\alpha \cdot \bar{\beta} \cdot \gamma]$. If $\widetilde{x}_{1}=\widetilde{x}_{0}$, then $\alpha$ can be taken to be the constant path at $x_{0}$.

Definition. We say that a map $\widehat{p}: \widehat{X} \rightarrow X$ has the unique path lifting property if whenever we are given two continuous maps $g_{1}, g_{2}:[0,1] \rightarrow \widehat{X}$ such that $\widehat{p} \circ g_{1}=\widehat{p} \circ g_{2}$ and $g_{1}(0)=g_{2}(0)$, we can conclude that $g_{1}=g_{2}$.

Although the construction of $\widetilde{X}$ appears to unwind every non-trivial loop of $X$, the resulting space $\widetilde{X}$ need not be simply-connected. The reason for this is that continuous paths in $\widetilde{X}$ might "jump between leaves". This rather counterintuitive phenomenon is exhibited by the following example.

EXAmple 2.7. This example is adapted from [28]. Subdivide the interior of an isosceles right triangle $T$ into infinitely many squares, accumulating along the hypotenuse, as shown in Figure 1 (left), change its embedding into $\mathbb{R}^{3}$ by elevating the centers of all squares to unit level as indicated and denote the resulting "bumpy triangle" by $B$. Finally, let $X$ either be the closure of $B$ in $\mathbb{R}^{3}$, as in Figure 1 (right), or let $X$ be the union of $B$ and the boundary of $T$. Then $X$ is either compact or locally path-connected, respectively.

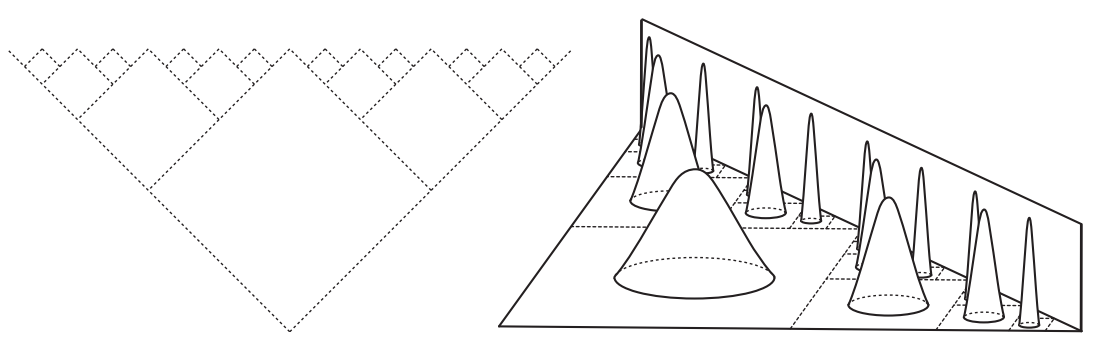

Fig. 1. A space permitting different lifts of the same path

We claim that, either way, $p: \widetilde{X} \rightarrow X$ does not have the unique path lifting property. Indeed, let $f:[0,1] \rightarrow X$ be the path which runs along 
the hypotenuse of our triangle with unit speed from left to right. There is, of course, the standard lift $g_{1}:[0,1] \rightarrow \widetilde{X}$ given by $g_{1}(s)=\left[f_{s}\right]$, where $f_{s}(t)=f(s t)$. However, another continuous lift $g_{2}:[0,1] \rightarrow \widetilde{X}$ is given by $g_{2}(t)=\left[\alpha_{t}\right]$, where $\left[\alpha_{t}\right]$ is the unique homotopy class of a path $\alpha_{t}$ which begins at the upper left corner of our triangle, goes straight to the bottom vertex, and then increases back up to $f(t)$, only using the dotted boundary lines of our squares. While $p \circ g_{1}=p \circ g_{2}=f$ and $g_{1}(0)=g_{2}(0)$, we have $g_{1}(t) \neq g_{2}(t)$ for all $t>0$. Hence, $\widetilde{X}$ is not simply-connected by the following lemma.

LEMma 2.8. $p: \widetilde{X} \rightarrow X$ has the unique path lifting property if and only if $\widetilde{X}$ is simply-connected.

Proof. Suppose that $p: \widetilde{X} \rightarrow X$ has the unique path lifting property. Recall that $\widetilde{x}_{0}=[\delta] \in \widetilde{X}$, where $\delta:[0,1] \rightarrow X$ is given by $\delta(t)=x_{0}$ for all $t \in[0,1]$, and that $p\left(\widetilde{x}_{0}\right)=x_{0}$. Let $h:[0,1] \rightarrow \widetilde{X}$ be a continuous map with $h(0)=h(1)=\widetilde{x}_{0}$. Define $f=p \circ h$ and let $g:([0,1], 0) \rightarrow\left(\widetilde{X}, \widetilde{x}_{0}\right)$ be the standard lift of $f:([0,1], 0) \rightarrow\left(X, x_{0}\right)$.

By assumption, we have $g=h$. In particular, $[f]=g(1)=h(1)=$ $h(0)=[\delta]$. Hence, there exists a continuous homotopy $H:[0,1] \times[0,1] \rightarrow X$ which contracts $f:[0,1] \rightarrow X$ to $x_{0}$ relative to its endpoints, that is, such that $H(\{0,1\} \times[0,1])=H([0,1] \times\{1\})=\left\{x_{0}\right\}$ and $H(t, 0)=f(t)$ for all $t \in[0,1]$. By Lemma 2.4, there is a continuous map $\widetilde{H}:[0,1] \times[0,1] \rightarrow \widetilde{X}$ with $\widetilde{H}(0,0)=\widetilde{x}_{0}$ and $H=p \circ \widetilde{H}$. Since the map $q:[0,1] \rightarrow \widetilde{X}$ given by $q(t)=\widetilde{H}(0, t)$ is such that $q(0)=\widetilde{x}_{0}$ and $p \circ q(t)=H(0, t)=x_{0}$ for all $t \in[0,1]$, then $q(t)=\widetilde{x}_{0}$ for all $t \in[0,1]$, by the assumed uniqueness of path lifting. Hence, $\widetilde{H}(0, t)=\widetilde{x}_{0}$ for all $t \in[0,1]$. Similarly, $\widetilde{H}(t, 1)=$ $\widetilde{H}(1, t)=\widetilde{x}_{0}$ for all $t \in[0,1]$. Finally, $h(t)=\widetilde{H}(t, 0)$ for all $t \in[0,1]$, since both are continuous lifts of $f:[0,1] \rightarrow X$ with $h(0)=\widetilde{x}_{0}=\widetilde{H}(0,0)$. Hence, $[h]=1 \in \pi_{1}\left(\tilde{X}, \widetilde{x}_{0}\right)$. We conclude that $\widetilde{X}$ is simply-connected.

Now suppose that $\widetilde{X}$ is simply-connected. Let $g_{1}, g_{2}:[0,1] \rightarrow \widetilde{X}$ be two continuous maps with $p \circ g_{1}=p \circ g_{2}$ and $g_{1}(0)=g_{2}(0)$. It will suffice to prove that $g_{1}(1)=g_{2}(1)$. Say, $g_{1}(0)=g_{2}(0)=[\alpha], g_{1}(1)=[\beta]$, and $g_{2}(1)=[\gamma]$. Let $u, v:([0,1], 0) \rightarrow\left(\widetilde{X}, \widetilde{x}_{0}\right)$ be the standard lifts of $\beta, \gamma:([0,1], 0) \rightarrow\left(X, x_{0}\right)$, respectively. Then $u(1)=[\beta]=g_{1}(1)$ and $v(1)=[\gamma]=g_{2}(1)$. If we define $\bar{g}_{i}(t)=g_{i}(1-t)$, then $\left[u \cdot \bar{g}_{1}\right]=\left[v \cdot \bar{g}_{2}\right]$, since $\widetilde{X}$ is simply-connected. Applying $p_{\#}$ to both sides of the previous equation, we get $[\beta \cdot \bar{f}]=[\gamma \cdot \bar{f}]$ (where $f=$ $\left.p \circ g_{1}=p \circ g_{2}\right)$, so that $[\beta]=[\gamma]$. Consequently, $g_{1}(1)=[\beta]=[\gamma]=g_{2}(1)$.

Next, we address metrizability issues.

Definition 2.9. We call $X$ homotopically Hausdorff at the point $x \in X$ if for every $g \in \pi_{1}(X, x) \backslash\{1\}$ there is an open subset $U \subseteq X$ with $x \in U$ such 
that there is no loop $\delta:\left(S^{1}, *\right) \rightarrow(U, x)$ with $[\delta]=g$. If $X$ is homotopically Hausdorff at every one of its points, then $X$ is said to be homotopically Hausdorff.

This useful notion, which we have just defined, already appears in [28] and [3]. We have adopted the terminology from [5], which is motivated by the following lemma.

Lemma 2.10. Suppose $X$ is Hausdorff. Then $\tilde{X}$ is Hausdorff if and only if $X$ is homotopically Hausdorff.

Proof. Suppose $X$ is homotopically Hausdorff. Let $[\alpha],[\beta] \in \widetilde{X}$ with $[\alpha] \neq[\beta]$. We wish to find open subsets $U, V \subseteq X$ such that $[\alpha] \in B([\alpha], U)$, $[\beta] \in B([\beta], V)$ and $B([\alpha], U) \cap B([\beta], V)=\emptyset$. If $\alpha(1) \neq \beta(1)$, it clearly suffices to take $U$ and $V$ such that $\alpha(1) \in U, \beta(1) \in V$ and $U \cap V=\emptyset$. If $\alpha(1)=\beta(1)$, then $[\bar{\alpha} \cdot \beta] \in \pi_{1}(X, \alpha(1)) \backslash\{1\}$, so that we can take $U=V$ with $\alpha(1) \in U$ such that there is no loop $\delta:\left(S^{1}, *\right) \rightarrow(U, \alpha(1))$ with $[\delta]=[\bar{\alpha} \cdot \beta]$. This implies that $[\beta] \notin B([\alpha], U)$ so that $B([\beta], U) \cap B([\alpha], U)=\emptyset$. We conclude that $\widetilde{X}$ is Hausdorff.

Conversely, if $X$ is not homotopically Hausdorff, then the proof of Lemma 2.11 below shows how to find distinct elements $\widetilde{x}_{1}$ and $\widetilde{x}_{2}$ of $\widetilde{X}$ which do not have disjoint neighborhoods.

The property of being homotopically Hausdorff is a necessary condition in our quest for a generalized universal covering:

Lemma 2.11. If $p: \widetilde{X} \rightarrow X$ has the unique path lifting property, then $X$ is homotopically Hausdorff.

Proof. Suppose, to the contrary, that there is an $x \in X$ and a $[\gamma] \in$ $\pi_{1}(X, x) \backslash\{1\}$ such that for every open subset $U \subseteq X$ with $x \in U$, there is a continuous map $\gamma^{\prime}:[0,1] \rightarrow U$ such that $\gamma^{\prime}(0)=\gamma^{\prime}(1)=x$ and $[\gamma]=\left[\gamma^{\prime}\right]$. Choose $[\alpha] \in \widetilde{X}$ with $p([\alpha])=x$. Put $\widetilde{x}_{1}=[\alpha]$ and $\widetilde{x}_{2}=[\alpha \cdot \gamma]$. Then $p\left(\widetilde{x}_{1}\right)=p\left(\widetilde{x}_{2}\right)=x$ and $\widetilde{x}_{1} \neq \widetilde{x}_{2}$. Let $U$ be an open subset of $X$ with $x \in U$. Choose $\gamma^{\prime}:[0,1] \rightarrow U$ such that $\gamma^{\prime}(0)=\gamma^{\prime}(1)=x$ and $\left[\gamma^{\prime}\right]=[\gamma]$. Then $\widetilde{x}_{2}=[\alpha \cdot \gamma]=\left[\alpha \cdot \gamma^{\prime}\right] \in B([\alpha], U)$ and $\widetilde{x}_{1}=[\alpha]=\left[\alpha \cdot \gamma^{\prime} \cdot \bar{\gamma}^{\prime}\right] \in B\left(\left[\alpha \cdot \gamma^{\prime}\right], U\right)$. That is, $\widetilde{x}_{1} \in B\left(\widetilde{x}_{2}, U\right)$ and $\widetilde{x}_{2} \in B\left(\widetilde{x}_{1}, U\right)$. Hence any function $g:[0,1] \rightarrow \widetilde{X}$ with $g(t) \in\left\{\widetilde{x}_{1}, \widetilde{x}_{2}\right\}$ will be continuous and such that $p \circ g(t)=x$ for all $t \in[0,1]$. This contradicts the unique path lifting property of $p: \widetilde{X} \rightarrow X$.

Lemma 2.12. Suppose $X$ is metrizable. Then $\widetilde{X}$ is metrizable if and only if $X$ is homotopically Hausdorff; in which case there is a metric $\widetilde{d}$ on $\widetilde{X}$, inducing the given topology, with respect to which the natural action of $\pi_{1}\left(X, x_{0}\right)$ on $\widetilde{X}$ is by isometry.

Proof. Suppose $(X, d)$ is a metric space and homotopically Hausdorff. Following [3], for $[\alpha],[\beta] \in \widetilde{X}$, we declare $\widetilde{d}([\alpha],[\beta])$ to be the greatest lower 
bound of the set $\{\operatorname{diam} \gamma([0,1]) \mid \gamma:[0,1] \rightarrow X$ continuous, $[\gamma]=[\bar{\alpha} \cdot \beta]\}$. Symmetry is built into this formula and the triangle inequality is verified easily. In order to show that $\widetilde{d}$ defines a metric on $\widetilde{X}$, we suppose that $\widetilde{d}([\alpha],[\beta])=0$ for some $[\alpha],[\beta] \in \widetilde{X}$. This forces $d(\alpha(1), \beta(1))=0$ so that $\alpha(1)=\beta(1)$. Put $x=\alpha(1)$. Let any $\varepsilon>0$ be given and consider $N(x, \varepsilon)=$ $\{y \in X \mid d(x, y)<\varepsilon\}$. Since $\widetilde{d}([\alpha],[\beta])<\varepsilon$, there is a loop $\gamma:\left(S^{1}, *\right) \rightarrow$ $(N(x, \varepsilon), x)$ with $[\gamma]=[\bar{\alpha} \cdot \beta]$. Since $X$ is homotopically Hausdorff at $x$, we conclude that $[\bar{\alpha} \cdot \beta]=1$, that is, $[\alpha]=[\beta]$.

The fact that $\widetilde{d}$ induces the correct topology on $\widetilde{X}$ follows from the inclusions

$$
\widetilde{N}([\alpha], \varepsilon) \subseteq B([\alpha], N(\alpha(1), \varepsilon)) \subseteq \widetilde{N}([\alpha], 2 \varepsilon),
$$

where $\tilde{N}([\alpha], \varepsilon)=\{[\beta] \in \widetilde{X} \mid \widetilde{d}([\alpha],[\beta])<\varepsilon\}$. Finally, since the natural action of $\pi_{1}\left(X, x_{0}\right)$ on $\tilde{X}$ is given by $[\eta] \cdot[\alpha]=[\eta \cdot \alpha]$, the very definition of $\widetilde{d}$ implies that $\widetilde{d}([\eta] \cdot[\alpha],[\eta] .[\beta])=\widetilde{d}([\alpha],[\beta])$ for all $[\alpha],[\beta] \in \widetilde{X}$ and $[\eta] \in$ $\pi_{1}\left(X, x_{0}\right)$.

REMARK 2.13 (The uniqueness of lifts). Suppose a map $\widehat{p}: \widehat{X} \rightarrow X$ has the unique path lifting property. Let $Y$ be any path-connected space, $f:(Y, y) \rightarrow(X, x)$ any continuous map, and $\widehat{x} \in \widehat{X}$ with $\widehat{p}(\widehat{x})=x$. If there exists a continuous map $g:(Y, y) \rightarrow(\widehat{X}, \widehat{x})$ such that $\widehat{p} \circ g=f$, then it is unique.

We summarize all of these observations in the following proposition.

Proposition 2.14. Suppose $p: \widetilde{X} \rightarrow X$ has the unique path lifting property. Then

(a) The map $p: \widetilde{X} \rightarrow X$ satisfies Properties $\mathrm{U}_{1}, \mathrm{U}_{2}, \mathrm{U}_{3}$ and $\mathrm{U}_{4}$.

(b) If $X$ is locally path-connected, then $p: \widetilde{X} \rightarrow X$ also satisfies $\mathrm{U}_{5}$.

(c) If $X$ is Hausdorff or metrizable, then so is $\widetilde{X}$ (with $\pi_{1}\left(X, x_{0}\right)$ acting by isometry).

3. The first shape homotopy group. We briefly recall the definition of the first shape homotopy group $\check{\pi}_{1}\left(X, x_{0}\right)$ of $X$ at $x_{0}$ from [21]. For every open cover $\mathcal{U}$ of $X$, designate one element $* \in \mathcal{U}$ with $x_{0} \in *$. Let $\mathcal{C}$ be the collection of all pointed normal covers $(\mathcal{U}, *)$ of $X$. (Recall that a normal cover $\mathcal{U}$ of $X$ is an open cover of $X$ which admits a partition of unity subordinated to $\mathcal{U}$. This partition of unity can always be chosen to be locally finite.) Then $\mathcal{C}$ is naturally directed by refinement. Denote by $(N(\mathcal{U}), *)$ a geometric realization of the pointed nerve of $\mathcal{U}$, that is, a geometric realization of the abstract simplicial complex $\left\{\Delta \mid \emptyset \neq \Delta \subseteq \mathcal{U}, \bigcap_{U \in \Delta} U \neq \emptyset\right\}$ with distinguished vertex $*$. For every $(\mathcal{U}, *),(\mathcal{V}, *) \in \mathcal{C}$ such that $(\mathcal{V}, *)$ refines $(\mathcal{U}, *)$, choose a pointed simplicial map puV $:(N(\mathcal{V}), *) \rightarrow(N(\mathcal{U}), *)$ 
with the property that the vertex corresponding to an element $V \in \mathcal{V}$ gets mapped to a vertex corresponding to an element $U \in \mathcal{U}$ with $V \subseteq U$. (Any assignment on the vertices which is induced by the refinement property will extend linearly.) Then $p_{\mathcal{U V}}$ is unique up to pointed homotopy and we denote its pointed homotopy class by $\left[p_{\mathcal{U} \mathcal{V}}\right]$. For each $(\mathcal{U}, *) \in \mathcal{C}$ choose a pointed map $p_{\mathcal{U}}:\left(X, x_{0}\right) \rightarrow(N(\mathcal{U}), *)$ such that $p_{\mathcal{U}}^{-1}(\operatorname{St}(U, N(\mathcal{U}))) \subseteq U$ for all $U \in \mathcal{U}$, where $\operatorname{St}(U, N(\mathcal{U}))$ denotes the open star of the vertex of $N(\mathcal{U})$ which corresponds to $U$. (For example, define $p_{\mathcal{U}}$ based on a locally finite partition of unity subordinated to $\mathcal{U}$.) Again, such a map $p_{\mathcal{U}}$ is unique up to pointed homotopy and we denote its pointed homotopy class by $\left[p_{\mathcal{U}}\right]$. Then $\left[p_{\mathcal{U V}} \circ p_{\mathcal{V}}\right]=\left[p_{\mathcal{U}}\right]$. The so-called (pointed) Čech expansion

$$
\left(X, x_{0}\right) \stackrel{\left(\left[p_{\mathcal{U}}\right]\right)}{\longrightarrow}\left((N(\mathcal{U}), *),\left[p_{\mathcal{U}}\right], \mathcal{C}\right)
$$

is an $\mathrm{HPol}_{*}$-expansion, so that we can define the first shape homotopy group of $X$, based at $x_{0}$, by

$$
\check{\pi}_{1}\left(X, x_{0}\right)=\varliminf_{\lim }\left(\pi_{1}(N(\mathcal{U}), *), p_{\mathcal{U V} \#}, \mathcal{C}\right) .
$$

Since the maps $p_{\mathcal{U}}$ induce homomorphisms $p_{\mathcal{U} \#}: \pi_{1}\left(X, x_{0}\right) \rightarrow \pi_{1}(N(\mathcal{U}), *)$ such that $p_{\mathcal{U} \#}=p_{\mathcal{U V} \#} \circ p_{\mathcal{V} \#}$ whenever $(\mathcal{V}, *)$ refines $(\mathcal{U}, *)$, we obtain an induced homomorphism

$$
\varphi: \pi_{1}\left(X, x_{0}\right) \rightarrow \check{\pi}_{1}\left(X, x_{0}\right)
$$

given by $\varphi([\alpha])=\left(\left[\alpha_{\mathcal{U}}\right]\right)$ where $\alpha_{\mathcal{U}}=p_{\mathcal{U}} \circ \alpha$.

4. Existence of generalized universal coverings. In search of criteria which would guarantee the existence of a generalized universal covering, we take our clues from the following theorem.

TheOrem 4.1. Suppose $X$ is paracompact Hausdorff. If $X$ is locally path-connected and semilocally simply-connected, then $\varphi: \pi_{1}\left(X, x_{0}\right) \rightarrow$ $\check{\pi}_{1}\left(X, x_{0}\right)$ is an isomorphism. If , in addition, $X$ is second countable, then $\pi_{1}\left(X, x_{0}\right)$ is countable.

REMARK. For a proof of the first assertion of Theorem 4.1 in the compact metric case or in the locally simply-connected case see [18] or [17], respectively. The second assertion is well known, but it also follows from our proof below.

Proof. Since $X$ is assumed to be paracompact Hausdorff, every open cover of $X$ is normal. It therefore suffices to show that every open cover $\mathcal{U}$ of $X$ is refined by an open cover $\mathcal{V}$ of $X$ such that $p_{\mathcal{V} \#}: \pi_{1}\left(X, x_{0}\right) \rightarrow$ $\pi_{1}(N(\mathcal{V}), *)$ is an isomorphism.

Let $\mathcal{U}$ be any open cover of $X$. As $X$ is locally path-connected and semilocally simply-connected, there is a cover $\mathcal{W}$ of $X$ by open path-connected 
sets which refines $\mathcal{U}$ and is such that for every $W \in \mathcal{W}, \pi_{1}(W) \rightarrow \pi_{1}(X)$ is trivial. Since $\mathcal{W}$ is a normal cover of $X$, there is an open cover $\mathcal{W}^{\prime}$ of $X$ which star-refines $\mathcal{W}$ [1, Definition 1.14 and Theorem 10.10]. That is, for every $W_{1}^{\prime} \in \mathcal{W}^{\prime}$ there is a $W \in \mathcal{W}$ such that for every $W_{2}^{\prime} \in \mathcal{W}^{\prime}$ with $W_{1}^{\prime} \cap W_{2}^{\prime} \neq \emptyset$ we have $W_{2}^{\prime} \subseteq W$. Since $X$ is locally path-connected, there is a cover $\mathcal{V}$ of $X$ by path-connected open sets which refines $\mathcal{W}^{\prime}$. (If $X$ is second countable, we can take $\mathcal{V}$ to be countable.) We conclude that $\mathcal{V}$ is a cover of $X$ by open path-connected sets such that every loop which lies in the union of any two elements of $\mathcal{V}$ is contractible in $X$. Therefore, $p_{\mathcal{V} \#}: \pi_{1}\left(X, x_{0}\right) \rightarrow \pi_{1}(N(\mathcal{V}), *)$ is an isomorphism [4, pp. 269-271]. Since $\mathcal{V}$ refines $\mathcal{U}$, the theorem follows.

REMARK 4.2. Even if $X$ is locally path-connected and $\varphi: \pi_{1}\left(X, x_{0}\right) \rightarrow$ $\check{\pi}_{1}\left(X, x_{0}\right)$ is an isomorphism, $X$ need not be semilocally simply-connected at any one of its points. Such is the case with the countable product $X=$ $X_{1} \times X_{2} \times \cdots$ of circles $X_{i}=S^{1}$. (Notice that the inverse sequence $X_{1} \leftarrow$ $X_{1} \times X_{2} \leftarrow X_{1} \times X_{2} \times X_{3} \leftarrow \cdots$ of compact ANR's and bonding maps which "forget" the last coordinate has limit $X$. It therefore yields an alternative $\mathrm{HPol}_{*}$ expansion for $X\left[21\right.$, Ch. I, §5]. Consequently, $\varphi: \pi_{1}(X, *) \rightarrow \check{\pi}_{1}(X, *)$ reduces to the standard isomorphism between $\pi_{1}\left(\prod_{i} X_{i}, *\right)$ and $\prod_{i} \pi_{1}\left(X_{i}, *\right)$. It also follows from this isomorphism that, for any open intervals $I_{i}$ of $X_{i}$, the inclusions $I_{1} \times \cdots \times I_{i} \times X_{i+1} \times X_{i+2} \times \cdots \hookrightarrow X$ do not induce trivial maps on $\pi_{1}$. Hence $X$ is not semilocally simply-connected at any point.)

However, if $p_{\mathcal{U} \#}: \pi_{1}\left(X, x_{0}\right) \rightarrow \pi_{1}(N(\mathcal{U}), *)$ is an isomorphism for some normal open cover $\mathcal{U}$ of $X$, then $X$ is semilocally simply-connected (cf. proof of Proposition 4.8).

Spaces with countable fundamental group. Recall that a necessary condition for $p: \widetilde{X} \rightarrow X$ to have the unique path lifting property is that $X$ be homotopically Hausdorff. In the case of a countable fundamental group, this condition is also sufficient:

Proposition 4.3. If $X$ is homotopically Hausdorff and $\pi_{1}\left(X, x_{0}\right)$ is countable, then $p: \widetilde{X} \rightarrow X$ has the unique path lifting property.

Proof. Let $g_{1}, g_{2}:[0,1] \rightarrow \widetilde{X}$ be continuous with $p \circ g_{1}=p \circ g_{2}$. For every $t \in[0,1]$, let $\alpha_{t}, \beta_{t}:([0,1], 0) \rightarrow\left(X, x_{0}\right)$ be continuous maps with $g_{1}(t)=\left[\alpha_{t}\right]$ and $g_{2}(t)=\left[\beta_{t}\right]$.

We claim that the function $f:[0,1] \rightarrow \pi_{1}\left(X, x_{0}\right)$ given by $f(t)=\left[\alpha_{t} \cdot \bar{\beta}_{t}\right]$ is continuous provided $\pi_{1}\left(X, x_{0}\right)$ is given the finite complement topology. First observe that $\alpha_{t}(1)=p\left(\left[\alpha_{t}\right]\right)=p \circ g_{1}(t)=p \circ g_{2}(t)=p\left(\left[\beta_{t}\right]\right)=\beta_{t}(1)$ for all $t \in[0,1]$, so that $f$ is well defined. Now, let $s \in[0,1]$ and let a finite subset $K \subseteq \pi_{1}\left(X, x_{0}\right)$ be given with $f(s) \in \pi_{1}\left(X, x_{0}\right) \backslash K$. Put $x=\alpha_{s}(1)$. Define an isomorphism $\varrho: \pi_{1}\left(X, x_{0}\right) \rightarrow \pi_{1}(X, x)$ by the formula $\varrho([\gamma])=\left[\bar{\alpha}_{s} \cdot \gamma \cdot \alpha_{s}\right]$. 
Since $X$ is homotopically Hausdorff at $x$ and since $K$ is finite, we may choose an open subset $U$ of $X$ such that there is no loop $\eta:\left(S^{1}, *\right) \rightarrow(U, x)$ with $[\eta]=\varrho\left(k f(s)^{-1}\right)$ and $k \in K$. By continuity of $g_{1}$ and $g_{2}$, we may choose a $\delta>0$ such that $\left[\alpha_{t}\right] \in B\left(\left[\alpha_{s}\right], U\right)$ and $\left[\beta_{t}\right] \in B\left(\left[\beta_{s}\right], U\right)$ for all $t \in[0,1]$ with $|s-t|<\delta$. Now let $t \in[0,1]$ with $|s-t|<\delta$. Then $\left[\alpha_{t}\right]=\left[\alpha_{s} \cdot \tau\right]$ and $\left[\beta_{t}\right]=\left[\beta_{s} \cdot \sigma\right]$ for some continuous maps $\tau, \sigma:[0,1] \rightarrow U$. So, $\eta=\tau \cdot \bar{\sigma}$ is a loop in $U$ based at $x$ with $[\eta]=[\tau \cdot \bar{\sigma}]=\left[\bar{\alpha}_{s} \cdot \alpha_{t} \cdot \bar{\beta}_{t} \cdot \beta_{s}\right]=\varrho\left(f(t) f(s)^{-1}\right)$. Therefore, $f(t) \in \pi_{1}\left(X, x_{0}\right) \backslash K$. This proves the claim.

Since the finite complement topology satisfies the $\mathrm{T}_{1}$-axiom, in order to finish the proof, it suffices to show that every path-component of a countable $\mathrm{T}_{1}$-space is a singleton. Suppose, to the contrary, that there is a non-constant continuous function $f:[0,1] \rightarrow \mathbb{N}$ into the natural numbers $\mathbb{N}$, endowed with some $\mathrm{T}_{1}$-topology. For each $n \in \mathbb{N}$, define $A_{n}=f^{-1}(\{n\})$. Since $\mathbb{N}$ is a $T_{1}$-space and $f$ is continuous, $\left\{A_{n} \mid n \in \mathbb{N}\right\}$ is a countable collection of pairwise disjoint closed subsets of $[0,1]$, at least two of which are non-empty, with the property that $[0,1]=\bigcup_{n \in \mathbb{N}} A_{n}$. It is an elementary fact of point-set topology that this is impossible.

Combining Proposition 4.3 with Proposition 2.14, we obtain

TheOREM 4.4. Suppose $X$ is homotopically Hausdorff and $\pi_{1}\left(X, x_{0}\right)$ is countable. Then $p: \widetilde{X} \rightarrow X$ is a generalized universal covering. If $X$ is also locally path-connected, then $p: \widetilde{X} \rightarrow X$ is an open map. If $X$ is Hausdorff, then so is $\widetilde{X}$.

REMARK 4.5. If $X$ is first countable, locally path-connected, homotopically Hausdorff and $\pi_{1}\left(X, x_{0}\right)$ countable, then $p: \widetilde{X} \rightarrow X$ is actually a (classical) universal covering. This follows from Proposition 4.6 below, whose proof can be found in [6]. We include the short proof as a warm-up for its generalization, Proposition 6.6.

Proposition 4.6. Suppose that $X$ has a countable basis at $x_{0}$, that $X$ is homotopically Hausdorff at $x_{0}$, and that $X$ is not semilocally simplyconnected at $x_{0}$. Then the cardinality of $\pi_{1}\left(X, x_{0}\right)$ is at least that of the continuum.

Proof. As $X$ is not semilocally simply-connected at $x_{0}$ but homotopically Hausdorff at $x_{0}$, we can inductively find a countable basis $U_{1} \supseteq U_{2} \supseteq \cdots$ for the topology of $X$ at $x_{0}$ and continuous maps $\alpha_{i}:[0,1] \rightarrow U_{i}$ such that $\alpha_{i}(0)=\alpha_{i}(1)=x_{0}$ with the following properties: $\left[\alpha_{i}\right] \neq 1 \in \pi_{1}\left(X, x_{0}\right)$ and $\left[\alpha_{i}\right] \neq[\beta] \in \pi_{1}\left(X, x_{0}\right)$ for all continuous maps $\beta:[0,1] \rightarrow U_{i+1}$ with $\beta(0)=\beta(1)=x_{0}$. For each increasing sequence $i_{1}<i_{2}<\cdots$ of natural numbers we can now define a continuous loop $\alpha:[0,1] \rightarrow X$ by the formula $\alpha(t)=\alpha_{i_{k}}\left(2-2^{k}(1-t)\right)$ when $1-1 / 2^{k-1} \leq t \leq 1-1 / 2^{k}$ and $\alpha(1)=x_{0}$. Then distinct sequences $i_{1}<i_{2}<\cdots$ and $i_{1}^{\prime}<i_{2}^{\prime}<\cdots$ yield distinct elements 
$[\alpha]$ and $\left[\alpha^{\prime}\right]$ of $\pi_{1}\left(X, x_{0}\right)$, respectively. To see why, assume, without loss of generality, that the sequences disagree already in the first entry and that $i_{1}<i_{1}^{\prime}$. Then both $\left[\alpha_{i_{1}}\right]^{-1}[\alpha]$ and $\left[\alpha^{\prime}\right]$ can be represented by loops in $U_{i_{1}+1}$, but $\left[\alpha_{i_{1}}\right]$ cannot. Hence $[\alpha] \neq\left[\alpha^{\prime}\right]$ and the proposition is proved.

Fundamental groups of arbitrary cardinality. We now shift our attention to fundamental groups of arbitrary cardinality.

Definition. For an open cover $\mathcal{U}$ of $X$ let $\pi\left(\mathcal{U}, x_{0}\right)$ be the subgroup of $\pi_{1}\left(X, x_{0}\right)$ which is generated by all elements of the form $[\alpha \cdot \tau \cdot \bar{\alpha}]$, where $\tau$ : $[0,1] \rightarrow U$ is any loop in some $U \in \mathcal{U}$ and $[\alpha] \in \widetilde{X}$ with $\alpha(1)=\tau(0)=\tau(1)$.

Remark. The above definition of $\pi\left(\mathcal{U}, x_{0}\right)$ follows [25]. Observe that each $\pi\left(\mathcal{U}, x_{0}\right)$ is a normal subgroup of $\pi_{1}\left(X, x_{0}\right)$ and that $\pi\left(\mathcal{V}, x_{0}\right) \leq \pi\left(\mathcal{U}, x_{0}\right)$ whenever $\mathcal{V}$ is an open cover of $X$ which refines $\mathcal{U}$. If $\pi\left(\mathcal{U}, x_{0}\right)=1$ for some open cover $\mathcal{U}$ of $X$, then $X$ is semilocally simply-connected; the converse holds for locally path-connected $X$, but not in general (cf. Remark 4.9).

Proposition 4.7. If $\bigcap_{\mathcal{U} \in \mathcal{C}} \pi\left(\mathcal{U}, x_{0}\right)=1$ for some collection $\mathcal{C}$ of open covers of $X$, then $p: \widetilde{X} \rightarrow X$ has the unique path lifting property.

Proof. We refer the reader to the proof of Proposition 6.7 below, which establishes a more general result. The proof of Proposition 6.7 is easily adapted to a proof of Proposition 4.7 and can be read at this point, upon the following substitutions: replace $H$ by $\{1\}$ (so that $\left[\delta_{1}\right]=\left[\delta_{2}\right]=1$ ), replace $\widetilde{X} / H$ by $\widetilde{X}$, replace $H[\alpha]$ by $[\alpha]$ when $[\alpha] \in \widetilde{X}$, and replace $H B$ by $B$ when $B \subseteq \tilde{X}$.

Proposition 4.8. If $\mathcal{U}$ ranges over all open covers of $X$, then $\bigcap_{\mathcal{U}} \pi\left(\mathcal{U}, x_{0}\right)$ is contained in the kernel of $\varphi: \pi_{1}\left(X, x_{0}\right) \rightarrow \check{\pi}_{1}\left(X, x_{0}\right)$.

Proof. Let $[\alpha] \in \bigcap_{\mathcal{U}} \pi\left(\mathcal{U}, x_{0}\right)$. Let $\mathcal{U}$ be a normal cover of $X$. As in Section 3 , choose a map $p_{\mathcal{U}}:\left(X, x_{0}\right) \rightarrow(N(\mathcal{U}), *)$ such that $p_{\mathcal{U}}^{-1}(\operatorname{St}(U, N(\mathcal{U}))) \subseteq$ $U$ for all $U \in \mathcal{U}$. Then the collection $\mathcal{V}=\left\{p_{\mathcal{U}}^{-1}(\operatorname{St}(U, N(\mathcal{U}))) \mid U \in \mathcal{U}\right\}$ is an open cover of $X$ which refines $\mathcal{U}$. By assumption, $[\alpha] \in \pi\left(\mathcal{V}, x_{0}\right)$. Therefore, we can write $[\alpha]=\left[\alpha_{1} \tau_{1} \bar{\alpha}_{1}\right] \cdots\left[\alpha_{n} \tau_{n} \bar{\alpha}_{n}\right]$ with loops $\tau_{i}:[0,1] \rightarrow V_{i}$ in some $V_{i}=p_{\mathcal{U}}^{-1}\left(\operatorname{St}\left(U_{i}, N(\mathcal{U})\right)\right) \in \mathcal{V}$ and $\left[\alpha_{i}\right] \in \widetilde{X}$ with $\alpha_{i}(1)=\tau_{i}(0)=\tau_{i}(1)$. Since $p_{\mathcal{U}} \circ \tau_{i}$ is a loop in the open star of the vertex of $N(\mathcal{U})$ which corresponds to $U_{i}$, it can be homotoped to that vertex, for each $i$. Consequently, $p_{\mathcal{U} \#}([\alpha])=p_{\mathcal{U} \#}\left(\left[\alpha_{1} \tau_{1} \bar{\alpha}_{1}\right] \cdots\left[\alpha_{n} \tau_{n} \bar{\alpha}_{n}\right]\right)=p_{\mathcal{U} \#}\left(\left[\alpha_{1} \bar{\alpha}_{1}\right] \cdots\left[\alpha_{n} \bar{\alpha}_{n}\right]\right)=1$.

REMARK 4.9. We now present two examples, demonstrating that

(i) $\bigcap_{\mathcal{U}} \pi\left(\mathcal{U}, x_{0}\right)$ need not equal the kernel of $\varphi: \pi_{1}\left(X, x_{0}\right) \rightarrow \check{\pi}_{1}\left(X, x_{0}\right)$;

(ii) $X$ might be semilocally simply-connected while $\bigcap_{\mathcal{U}} \pi\left(\mathcal{U}, x_{0}\right) \neq 1$. 
Following [7], first consider

$$
\begin{aligned}
Y= & \left\{(x, y, z) \in \mathbb{R}^{3} \mid z=0,0 \leq x<1, y=\sin \left(\frac{1}{1-x}\right)\right\} \\
& \cup(\{1\} \times[-1,1] \times\{0\}) .
\end{aligned}
$$

Let $Z$ be the subset of $\mathbb{R}^{3}$ obtained by revolving $Y$ about the $y$-axis, with base point $x_{0}=(0, \sin 1,0)$. Choose any arc $a$ in $\mathbb{R}^{3}$ with $Z \cap a=\partial a=$ $\left\{x_{0},(1,1,0)\right\}$ and put $X=Z \cup a$. Clearly, for sufficiently small $\varepsilon>0$, any cover $\mathcal{U}$ of $X$ by open metric balls of radius $\varepsilon$ will have the property that $\pi\left(\mathcal{U}, x_{0}\right)=1$. Therefore, $\bigcap_{\mathcal{U}} \pi\left(\mathcal{U}, x_{0}\right)=1$.

The above example is an "inside-out" version of [11, Example 2.4], where the role of $Y$ is played by the union of the curve $0<x \leq 1, y=\sin 1 / x$ with $\{0\} \times[-1,1] \times\{0\}$ and a similar arc $a$ connects the two path-components. In each version of this example, both $\pi_{1}\left(X, x_{0}\right)$ and $\check{\pi}_{1}\left(X, x_{0}\right)$ are infinite cyclic, while the kernel of the homomorphism $\varphi: \pi_{1}\left(X, x_{0}\right) \rightarrow \check{\pi}_{1}\left(X, x_{0}\right)$ equals $\pi_{1}\left(X, x_{0}\right)$. Both examples are semilocally simply-connected. However, in the original example from [11], we have $\pi\left(\mathcal{U}, x_{0}\right)=\pi_{1}\left(X, x_{0}\right)$ for every open cover $\mathcal{U}$ of $X$, because the loop generating $\pi_{1}\left(X, x_{0}\right)$ can be homotoped (freely) arbitrarily closely to the origin.

We have arrived at the main theorem of this article:

Theorem 4.10. Suppose $\varphi: \pi_{1}\left(X, x_{0}\right) \rightarrow \check{\pi}_{1}\left(X, x_{0}\right)$ is injective. Then

(a) The map $p: \widetilde{X} \rightarrow X$ is a generalized universal covering.

(b) The group $G=\operatorname{Aut}(\widetilde{X} \stackrel{p}{\rightarrow} X)$ of covering transformations is isomorphic to $\pi_{1}\left(X, x_{0}\right)$ and acts freely and transitively on every fiber $p^{-1}(\{x\})$ with $x \in X$.

(c) If $X$ is locally path-connected, then $p: \widetilde{X} \rightarrow X$ is an open map and $\widetilde{X} / G \approx X$.

(d) If $X$ is Hausdorff or metrizable, then so is $\widetilde{X}$.

(e) If $X$ is metrizable, then there is a metric for $\widetilde{X}$ such that $G$ acts by isometry.

Proof. This follows from Propositions 4.8, 4.7 and 2.14.

EXAmple 4.11 (Manifolds and CW-complexes). If $X$ is locally pathconnected and semilocally simply-connected, then $X$ has a classical simply connected covering. Since this classical universal covering satisfies Properties $\mathrm{U}_{1}-\mathrm{U}_{3}$, it agrees with the generalized universal covering (see Section 1.1). For example, if $X$ is a manifold or a CW-complex, Theorem 4.10, whose assumption is satisfied by Theorem 4.1, reduces to the classical theory.

EXAMPLE 4.12 (The countably infinite product of circles). From Remark 4.2 we know that the countably infinite product of circles $X=S^{1} \times$ 
$S^{1} \times \cdots$ does not have a classical universal covering, although $\varphi: \pi_{1}\left(X, x_{0}\right)$ $\rightarrow \check{\pi}_{1}\left(X, x_{0}\right)$ is an isomorphism. However, by Theorem 4.10 , there is a metrizable generalized universal covering $p: \widetilde{X} \rightarrow X$ whose group of covering transformations is isomorphic to $\pi_{1}\left(X, x_{0}\right)$ and which acts by isometry with quotient $X$. This generalized universal covering is easily identified as the product $p=\left(p_{i}\right)_{i}$ of the classical universal coverings $p_{i}: \mathbb{R} \rightarrow S^{1}$, because $\left(p_{i}\right)_{i}: \prod_{i} \mathbb{R} \rightarrow \prod_{i} S^{1}$ clearly satisfies Properties $\mathrm{U}_{1}-\mathrm{U}_{3}$.

EXAMPLE 4.13 (Subsets of closed surfaces). It is proved in [12] that if $X$ is any subset of a closed surface, then $\varphi: \pi_{1}\left(X, x_{0}\right) \rightarrow \check{\pi}_{1}\left(X, x_{0}\right)$ is injective.

Remark (Topological $\mathbb{R}$-trees). The classical universal covering of a (connected) 1-dimensional simplicial complex is by a tree. Below, we will generalize this situation. The natural generalization of a tree is an $\mathbb{R}$-tree, that is, a metric space in which every pair of points is connected by a unique arc and that arc is the image of an isometric embedding of a closed interval of the real line.

In what follows, we are mainly interested in the topological properties of $\mathbb{R}$-trees, namely that they are uniquely arcwise connected, locally arcwise connected, metrizable, 1-dimensional and contractible. For reasons that will become apparent in Example 4.15, we often wish to de-emphasize the specific metric of the $\mathbb{R}$-tree.

Recall that every uniquely arcwise connected and locally arcwise connected metrizable space admits a compatible metric which makes it an $\mathbb{R}$ tree [24, Theorem 5.1]. We will therefore call a uniquely arcwise connected and locally arcwise connected metrizable space a topological $\mathbb{R}$-tree.

EXAMPLE 4.14 (1-dimensional path-connected separable metric spaces are covered by topological $\mathbb{R}$-trees). If $X$ is 1 -dimensional, compact and Hausdorff, or if $X$ is 1-dimensional, separable and metrizable, then $\varphi$ : $\pi_{1}\left(X, x_{0}\right) \rightarrow \check{\pi}_{1}\left(X, x_{0}\right)$ is injective by [9, Corollary 1.2 and Final Remark], so that we have a generalized universal covering $p: \widetilde{X} \rightarrow X$ by Theorem 4.10. We claim that, in either case, $\widetilde{X}$ is uniquely arcwise connected.

Since $\widetilde{X}$ is path-connected and Hausdorff, it is arcwise connected. Suppose, to the contrary, that $\widetilde{X}$ is not uniquely arcwise connected. Then $\widetilde{X}$ contains a simple closed curve $\widetilde{\alpha}: S^{1} \rightarrow \widetilde{X}$ which contracts in $\widetilde{X}$ by some map $\widetilde{f}: B^{2} \rightarrow \widetilde{X}$ such that $\widetilde{f} \circ i=\widetilde{\alpha}$, where $i: S^{1} \hookrightarrow B^{2}$ denotes inclusion. Consider the projections $\alpha=p \circ \widetilde{\alpha}: S^{1} \rightarrow X$ and $f=p \circ \widetilde{f}: B^{2} \rightarrow X$. Since $f\left(B^{2}\right)$ is a 1 -dimensional compact metric space, the map $f: B^{2} \rightarrow X$ factors through a dendrite, i.e. it factors through a compact, path-connected and locally path-connected metric space $D$ which does not contain any simple closed curve (see [8] or [5, Theorem 3.7]). Specifically, we have $f=g \circ h$ with maps $h: B^{2} \rightarrow D$ and $g: D \rightarrow X$. Considering the loop $h \circ i: S^{1} \rightarrow D$, 
there must be $x, y \in S^{1}$ and $d \in D$ such that $h \circ i(x)=d=h \circ i(y)$. Since dendrites are contractible, there is a lift $\widetilde{g}:(D, d) \rightarrow(\widetilde{X}, \widetilde{\alpha}(x))$ of $g:(D, d) \rightarrow(X, \alpha(x))$ with $p \circ \widetilde{g}=g$. Then $p \circ \widetilde{g} \circ h \circ i=g \circ h \circ i=f \circ i=\alpha$, so that $\widetilde{g} \circ h \circ i:\left(S^{1}, x\right) \rightarrow(\widetilde{X}, \widetilde{\alpha}(x))$ is another lift of $\alpha$, and hence equals $\widetilde{\alpha}$. Thus, $\widetilde{\alpha}(x)=\widetilde{g} \circ h \circ i(x)=\widetilde{g}(d)=\widetilde{g} \circ h \circ i(y)=\widetilde{\alpha}(y)$, contradicting the fact that $\widetilde{\alpha}$ is a simple closed curve.

If $X$ is 1-dimensional, separable and metrizable, it follows that $\widetilde{X}$ is a topological $\mathbb{R}$-tree. Note that the group of covering transformations $\pi_{1}\left(X, x_{0}\right)$ $\approx \operatorname{Aut}(\widetilde{X} \stackrel{p}{\rightarrow} X)$ acts on $\widetilde{X}$ by homeomorphism and that, by Theorem 4.10, we can metrize $\widetilde{X}$ so that this action is by isometry. However, as the next example shows, it might happen that no $\mathbb{R}$-tree metric of $\widetilde{X}$ allows for an action by isometry.

EXAmple 4.15 (The Hawaiian Earring). By the above, Theorem 4.10 applies to the Hawaiian Earring $\left(X, x_{0}\right)$ of Example 2.2. However, $p: \widetilde{X} \rightarrow$ $X$ is not a classical covering. Indeed, it is not even a Hurewicz fibration, since it does not have the homotopy lifting property with respect to every space: if $Y=p^{-1}\left(\left\{x_{0}\right\}\right) \subseteq \widetilde{X}$ is the fiber over the origin and $\alpha$ is a simple closed curve around any one of the loops of $X$ based at $x_{0}$, then the partial lift $g: Y \times\{0\} \rightarrow \widetilde{X}$ given by $g(y, 0)=y$ of $F: Y \times I \rightarrow X$ given by $F(y, t)=\alpha(t)$ cannot be extended to a full lift. (Otherwise, for every $0<t<1$, the map $h: Y \rightarrow \widetilde{X}$ given by $h(y)=g(y, t)$ would map the non-discrete fiber $Y$ homeomorphically onto the discrete fiber $p^{-1}(\{\alpha(t)\})$.)

While $\widetilde{X}$ is not a CW-complex, it is, by construction, the union of (uncountably many) open arcs and the set $Y$ of "branch points", which corresponds bijectively to $\pi_{1}\left(X, x_{0}\right)$. However, the action of $\operatorname{Aut}(\widetilde{X} \stackrel{p}{\rightarrow} X)$ on the $\mathbb{R}$-tree $\widetilde{X}$, when metrized by an $\mathbb{R}$-tree metric, cannot possibly be by isometry. For if $l_{n}:([0,1], 0) \rightarrow\left(X, x_{0}\right)$ is the simple closed loop given by $l_{n}(t)=\left(\frac{1}{n} \sin (2 \pi t), \frac{1}{n}-\frac{1}{n} \cos (2 \pi t)\right)$, then every lift $\widetilde{l}_{n}:([0,1], 0) \rightarrow(\widetilde{X}, \widetilde{x})$ of $l_{n}$ with $p(\widetilde{x})=x_{0}$ can be translated to any other such lift by an element of $\operatorname{Aut}(\widetilde{X} \stackrel{p}{\rightarrow} X)$. If all such lifts had the same length, we would be able to construct an arc $\widetilde{l}_{\infty}$ in $\widetilde{X}$ of infinite length by lifting the continuous function $l_{\infty}:([0,1], 0) \rightarrow\left(X, x_{0}\right)$ which iteratively traverses each $l_{n}$ a sufficiently large number of times, i.e., $l_{\infty}=\left(l_{1}\right)^{i_{1}}\left(l_{2}\right)^{i_{2}} \ldots$. Since the length of an arc in an $\mathbb{R}$-tree equals the distance between its endpoints, this is not possible.

As indicated in [27, Remark 3.8], it is possible to give an explicit combinatorial description of $\widetilde{X}$ and of the action of $\pi_{1}\left(X, x_{0}\right)$ on $\tilde{X}$. In [26, Theorem 1.12], the group $\pi_{1}\left(X, x_{0}\right)$ is described in terms of sequences of words in the letters $l_{1}^{ \pm 1}, l_{2}^{ \pm 1}, \ldots[26$, Definition 1.1], along with a set of rules for how to multiply these "word sequences", i.e., how to concatenate and cancel appropriately [26, Definition 1.11]. (This group should not be mistaken for 
the inverse limit of free groups [27, Definition 2.2], as was erroneously done in $[2, \S 8]$.) By construction, an element $\widetilde{x}$ of $\widetilde{X}$ can then be thought of as a triple $\left(\omega, l_{n}^{ \pm 1}, t\right)$, which indicates that $\widetilde{x}$ lies over the point $l_{n}^{ \pm 1}(t)$ of the loop $l_{n}:[0,1] \rightarrow X$ on the leaf corresponding to the word sequence $\omega$, where for the branch points we use $t=0$ but no specific second coordinate. In order to uniquely parametrize $\widetilde{X}$, only consider reduced triples $\left(\omega, l_{i}^{\varepsilon}, t\right)$, where $\omega$ does not have a "last letter" $l_{i}^{-\varepsilon}$, and identify each terminal point $\left(\omega, l_{i}^{\varepsilon}, 1\right)$ with every initial point of the form $\left(\omega \cdot l_{i}^{\varepsilon}, l_{j}^{\delta}, 0\right)$, for all permitted second coordinates. Then $p: \widetilde{X} \rightarrow X$ is given by $p\left(\omega, l_{n}^{\varepsilon}, t\right)=\left(\frac{\varepsilon}{n} \sin (2 \pi t), \frac{1}{n}-\frac{1}{n} \cos (2 \pi t)\right)$ and the action of $\pi_{1}\left(X, x_{0}\right)$ on $\widetilde{X}$ is given by $\eta \cdot\left(\omega, l_{n}^{\varepsilon}, t\right)=\left(\eta \cdot \omega \cdot l_{n}^{\varepsilon}, l_{n}^{-\varepsilon}, 1-t\right)$ if the product of the word sequences $\eta$ and $\omega$ has a last letter $l_{n}^{-\varepsilon}$, and $\eta \cdot\left(\omega, l_{n}^{\varepsilon}, t\right)=\left(\eta \cdot \omega, l_{n}^{\varepsilon}, t\right)$ otherwise.

EXAMPLE 4.16 (Trees of manifolds). If $X$ is the limit of an inverse system of closed PL-manifolds of some fixed dimension, whose consecutive terms are obtained by connect summing with closed PL-manifolds, which in turn are trivialized by the bonding maps, then $X$ is called a tree of manifolds. For example, the Pontryagin surface $\Pi_{2}$ is a tree of manifolds, namely a tree of real projective planes. Every tree of manifolds is pathconnected and locally path-connected, but it need not be semilocally simplyconnected at any one of its points. Trees of manifolds arise as boundaries of certain Coxeter groups and as boundaries of certain negatively curved geodesic spaces [11]. It is shown in [11] that if $X$ is a tree of manifolds, then $\varphi: \pi_{1}\left(X, x_{0}\right) \rightarrow \check{\pi}_{1}\left(X, x_{0}\right)$ is injective, provided the attachments are sufficiently dense in the case of surfaces (which they are for $\Pi_{2}$ ).

REMARK 4.17 (The non-locally path-connected case). For a non-locally path-connected space $X$, the quotient space $\widetilde{X} / G$ of $\widetilde{X}$ by the automorphism group $G=\operatorname{Aut}(\widetilde{X} \stackrel{p}{\rightarrow} X)$ is homeomorphic to its natural "local path-connectification": the smallest topology on $X$ which contains the given topology and is locally path-connected, that is, the topology which is generated by all path-components of all open subsets of $X$. For example, if $X$ is the suspension of $\{1,1 / 2,1 / 3, \ldots\} \cup\{0\} \subseteq \mathbb{R}$, then $\widetilde{X} / G$ is homeomorphic to the suspension of a discrete countable space.

5. First countable spaces. If $X$ is first countable, then Property $\mathrm{U}_{3}$ (as well as Property $\mathrm{R}_{3}$ of Section 6 ) reduces to the unique lifting of paths and their homotopies:

Proposition 5.1. Let $\widehat{X}$ be any topological space and $\widehat{p}: \widehat{X} \rightarrow X$ any continuous function. Suppose $\widehat{p}: \widehat{X} \rightarrow X$ has the homotopy lifting property with respect to points as well as $[0,1]$ and that $\widehat{p}: \widehat{X} \rightarrow X$ has the unique 
path lifting property. If $X$ is first countable, then $\widehat{p}: \widehat{X} \rightarrow X$ satisfies the following general lifting property:

For every path-connected and locally path-connected space $Y$, every continuous map $f:(Y, y) \rightarrow(X, x)$ and every $\widehat{x} \in \widehat{X}$ with $\widehat{p}(\widehat{x})=x$, there is a unique continuous function $g:(Y, y) \rightarrow(\widehat{X}, \widehat{x})$ with $\widehat{p} \circ g=f$, provided $f_{\#}\left(\pi_{1}(Y, y)\right) \leq \widehat{p}_{\#}\left(\pi_{1}(\widehat{X}, \widehat{x})\right)$.

REMARK. Note that every Hurewicz fibration $\widehat{p}: \widehat{X} \rightarrow X$ which has the unique path lifting property automatically satisfies this lifting property $[25$, Theorem 2.4.5].

Proof. Let $Y$ be path-connected and locally path-connected, $f:(Y, y) \rightarrow$ $(X, x)$ a continuous map with $f_{\#}\left(\pi_{1}(Y, y)\right) \leq \widehat{p}_{\#}\left(\pi_{1}(\widehat{X}, \widehat{x})\right)$, and let $\widehat{x} \in \widehat{X}$ be such that $\widehat{p}(\widehat{x})=x$. By Remark 2.13, we only have to prove the existence of $g$.

We define a function $g: Y \rightarrow \widehat{X}$ as follows: for $w \in Y$ choose any path $\alpha:[0,1] \rightarrow Y$ with $\alpha(0)=y$ and $\alpha(1)=w$. Let $\beta:[0,1] \rightarrow \widehat{X}$ be the unique continuous map with $f \circ \alpha=\widehat{p} \circ \beta$ and $\beta(0)=\widehat{x}$. Define $g(w)=\beta(1)$.

Since $f_{\#}\left(\pi_{1}(Y, y)\right) \leq \widehat{p}_{\#}\left(\pi_{1}(\widehat{X}, \widehat{x})\right)$, the assumed lifting properties of $\widehat{p}$ : $\widehat{X} \rightarrow X$ imply that the definition of $g$ does not depend on the choice of $\alpha$. By definition, $f(w)=f(\alpha(1))=\widehat{p}(\beta(1))=\widehat{p}(g(w))$ for all $w \in Y$, so that $f=\widehat{p} \circ g$.

In order to show that $g: Y \rightarrow \widehat{X}$ is continuous, let $w \in Y$ and an open subset $V \subseteq \widehat{X}$ with $g(w) \in V$ be given. Let $U_{1} \supseteq U_{2} \supseteq \cdots$ be a countable basis for the topology of $X$ at $f(w)$. By continuity of $f: Y \rightarrow X$, for each $i$, there is an open subset $W_{i} \subseteq Y$ with $w \in W_{i}$ and $f\left(W_{i}\right) \subseteq U_{i}$. By assumption, we may choose each $W_{i}$ path-connected. It suffices to prove that $g\left(W_{i}\right) \subseteq V$ for some $i$. Suppose, to the contrary, that for every $i$ there is a $w_{i} \in W_{i}$ with $g\left(w_{i}\right) \notin V$. For each $i$, choose a continuous path $\alpha_{i}:[0,1]$ $\rightarrow W_{i}$ with $\alpha_{i}(0)=w$ and $\alpha_{i}(1)=w_{i}$. Let $\beta_{i}:[0,1] \rightarrow \widehat{X}$ be the unique continuous map with $f \circ \alpha_{i}=\widehat{p} \circ \beta_{i}$ and $\beta_{i}(0)=g(w)$. Then $\beta_{i}(1)=g\left(w_{i}\right) \notin V$ for every $i$, by definition of $g: Y \rightarrow \widehat{X}$. Define a function $\alpha:[0,1] \rightarrow Y$ (not necessarily continuous at 1 ) which runs through $\alpha_{1}$ in $1 / 3$ of the time and then traverses $\alpha_{1}$ in reverse in $1 / 3$ of the time, then runs through $\alpha_{2}$ in $1 / 9$ of the time and then traverses $\alpha_{2}$ in reverse in $1 / 9$ of the time, etc. Specifically, for $1-3 / 3^{i} \leq t \leq 1-2 / 3^{i}$ put $\alpha(t)=\alpha_{i}\left(3^{i}(t-1)+3\right)$; for $1-2 / 3^{i} \leq t \leq 1-1 / 3^{i}$ put $\alpha(t)=\alpha_{i}\left(3^{i}(1-t)-1\right)$; and put $\alpha(1)=w$. Since $f \circ \alpha\left(\left[1-3 / 3^{i}, 1-1 / 3^{i}\right]\right)=f \circ \alpha_{i}([0,1]) \subseteq f\left(W_{i}\right) \subseteq U_{i}$, we see that $f \circ \alpha:[0,1] \rightarrow X$ is continuous. Let $\beta:[0,1] \rightarrow \widehat{X}$ be the unique continuous map with $f \circ \alpha=\widehat{p} \circ \beta$ and $\beta(0)=g(w) \in V$. By uniqueness of path lifting, we have $\beta\left(1-2 / 3^{i}\right)=\beta_{i}(1)=g\left(w_{i}\right) \notin V$ for all $i$. This contradicts the continuity of $\beta:[0,1] \rightarrow \widehat{X}$ at 1 . 
REMARK 5.2. It follows from the next proposition that, for locally pathconnected and first countable $X$, Properties $\mathrm{U}_{1}-\mathrm{U}_{3}$ imply Property $\mathrm{U}_{5}$.

Proposition 5.3. Let $\widehat{X}$ be any topological space and $\widehat{p}: \widehat{X} \rightarrow X$ any continuous function. Suppose $\widehat{p}: \widehat{X} \rightarrow X$ has the homotopy lifting property with respect to points. If $X$ is locally path-connected and first countable, then $\widehat{p}: \widehat{X} \rightarrow X$ is open.

Proof. Let $U$ be an open subset of $\widehat{X}$ and put $V=\widehat{p}(U)$. In order to show that $V$ is open in $X$, we let $x \in V$ and choose a countable basis $U_{1} \supseteq U_{2} \supseteq \cdots$ for the topology of $X$ at $x$ consisting of path-connected open sets. Choose $\widehat{x} \in U$ with $\widehat{p}(\widehat{x})=x$. It suffices to show that there is an $i$ with $U_{i} \subseteq V$. Suppose, to the contrary, that for each $i$ there is a $u_{i} \in U_{i} \backslash V$. For each $i$, choose a continuous path $\alpha_{i}:[0,1] \rightarrow U_{i}$ with $\alpha_{i}(0)=u_{i+1}$ and $\alpha_{i}(1)=u_{i}$. Define a function $\alpha:[0,1] \rightarrow X$ by $\alpha(t)=\alpha_{i}\left(2^{i} t-1\right)$ for $1 / 2^{i} \leq t \leq 1 / 2^{i-1}$ and put $\alpha(0)=x$. Since $\alpha\left(\left[1 / 2^{i}, 1 / 2^{i-1}\right]\right) \subseteq U_{i}$ for all $i$, $\alpha:[0,1] \rightarrow X$ is continuous. By assumption, there is a continuous map $\beta:[0,1] \rightarrow \widehat{X}$ such that $\alpha=\widehat{p} \circ \beta$ and $\beta(0)=\widehat{x} \in U$. Then $\widehat{p}\left(\beta\left(1 / 2^{i-1}\right)\right)=$ $\alpha\left(1 / 2^{i-1}\right)=u_{i} \notin V$ so that $\beta\left(1 / 2^{i-1}\right) \notin U$ for all $i$. This contradicts the continuity of $\beta$ at 0 .

REMARK 5.4. We cannot drop the assumption of first countability in Proposition 5.3. For example, let $X$ be a wedge of uncountably many circles joined at the common point $x_{0}$, with the CW-topology, and let $p: \widetilde{X} \rightarrow X$ be its (classical) universal covering. Let $X^{\prime}$ be the same point-set as $X$ but with slightly coarser topology: eliminate all those open subsets $U \subseteq X$ from the topology which contain $x_{0}$ but miss points from uncountably many circles. Then $p: \widetilde{X} \rightarrow X^{\prime}$ is still continuous. Observe that every sequentially compact subset of $X^{\prime}$ is contained in the union of finitely many circles. Consequently, a function $f:[0,1]^{n} \rightarrow X^{\prime}$ is continuous if and only if $f$ : $[0,1]^{n} \rightarrow X$ is continuous. It follows that $X^{\prime}$ is still locally path-connected and that $p: \widetilde{X} \rightarrow X^{\prime}$ is still a Serre fibration with the unique path lifting property. However, $p: \widetilde{X} \rightarrow X^{\prime}$ is no longer an open map: while $p: \widetilde{X} \rightarrow X$ is a local homeomorphism, the new map $p: \widetilde{X} \rightarrow X^{\prime}$ fails to map many elements of the local neighborhood system of a point in $p^{-1}\left(\left\{x_{0}\right\}\right)$ to open subsets of $X^{\prime}$.

6. Generalized regular coverings. By Lemma 2.6, every subgroup $H \leq \pi_{1}\left(X, x_{0}\right)$ is a subgroup of the automorphism group of $\widetilde{X} \stackrel{p}{\rightarrow} X$, where its natural action is given by $[\alpha] \cdot[\beta]=[\alpha \cdot \beta]$ for $[\alpha] \in H$ and $[\beta] \in \widetilde{X}$. We will denote by $\widetilde{X} / H$ the quotient space of $\widetilde{X}$ by the action of $H$, and use again the notation $\tilde{X} / H \stackrel{p}{\rightarrow} X$ for the continuous endpoint projection (which is a quotient map if and only if the endpoint projection $\widetilde{X} \rightarrow X$ is a quotient map). 
For $\widetilde{x} \in \widetilde{X}$, we will also use the notation $H \widetilde{x}=\{\widetilde{y} \in \widetilde{X} \mid \widetilde{y}=h . \widetilde{x}$ for some $h \in H\} \in \widetilde{X} / H$. Moreover, for $B \subseteq \widetilde{X}$, we will write $H B=\{H \widetilde{x} \mid \widetilde{x} \in B\}$ $\subseteq \widetilde{X} / H$.

Since a basis for the topology of $\widetilde{X}$ is given by $\{B([\alpha], U) \mid[\alpha] \in \widetilde{X}, U \subseteq$ $X$ open $\}$, the collection $\{H B([\alpha], U) \mid[\alpha] \in \widetilde{X}, U \subseteq X$ open $\}$ forms a basis for the quotient space $\widetilde{X} / H$. We also note that if $H[\beta] \in H B([\alpha], U)$, then $H B([\beta], U)=H B([\alpha], U)$.

Recall that, regarding the map $p: \widetilde{X} / H \rightarrow X$, we have the following classical theorem [25, Theorem 2.5.13].

TheORem 6.1. Suppose $X$ is locally path-connected. Let $\mathcal{U}$ be any open cover of $X$. If $\pi\left(\mathcal{U}, x_{0}\right) \leq H \leq \pi_{1}\left(X, x_{0}\right)$, then $p: \widetilde{X} / H \rightarrow X$ is a (classical) covering space.

The general situation is more complicated, as the next example illustrates.

Example 6.2. Let $X$ be the Hawaiian Earring and let $H$ be the (free) subgroup of $\pi_{1}\left(X, x_{0}\right)$ which is generated by $\left\{\left[l_{n}\right] \mid n \in \mathbb{N}\right\}$, where $l_{n}$ is the simple closed loop of $X$ of radius $1 / n$ based at $x_{0}$. Then $p: \widetilde{X} / H \rightarrow X$ does not have the unique path lifting property, although $p: \widetilde{X} \rightarrow X$ does. To see why, define a continuous loop $f:[0,1] \rightarrow X$ as follows: let $C \subseteq[0,1]$ be the standard middle third Cantor set. For each component $(a, b)$ of $[0,1] \backslash C$ with $b-a=1 / 3^{n}$ and $t \in[a, b]$ define $f(t)=l_{n}\left(\frac{t-a}{b-a}\right)$ and put $f(t)=x_{0}$ for $t \in C$. We will construct two different lifts of $f$.

Let $g_{1}:([0,1], 0) \rightarrow\left(\widetilde{X} / H, H \widetilde{x}_{0}\right)$ be the standard lift of $f:([0,1], 0) \rightarrow$ $\left(X, x_{0}\right)$ to $\left(\widetilde{X}, \widetilde{x}_{0}\right)$ followed by the quotient map $\widetilde{X} \rightarrow \widetilde{X} / H$, so that $f=$ $p \circ g_{1}$. Specifically, $g_{1}(t)=H\left[f \circ \tau_{t}\right]$, where $\tau_{t}:[0,1] \rightarrow[0, t]$ is defined by $\tau_{t}(s)=t s$.

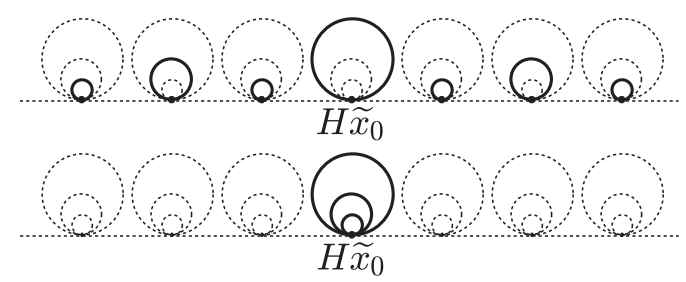

Fig. 2. Two different lifts of $f:[0,1] \rightarrow X$ to $\tilde{X} / H$

A second continuous lift $g_{2}:([0,1], 0) \rightarrow\left(\widetilde{X} / H, H \widetilde{x}_{0}\right)$ with $f=p \circ g_{2}$ can be constructed by lifting the loops individually to the same base point. Specifically, let $L_{n}:([0,1], 0) \rightarrow\left(\widetilde{X} / H, H \widetilde{x}_{0}\right)$ be the standard lift of $l_{n}$ : $([0,1], 0) \rightarrow\left(X, x_{0}\right)$ to $\left(\widetilde{X}, \widetilde{x}_{0}\right)$ followed by the quotient map $\widetilde{X} \rightarrow \widetilde{X} / H$. Then $L_{n}(1)=H\left[l_{n} \circ \tau_{1}\right]=H\left[l_{n}\right]=H \widetilde{x}_{0}$. So, each $L_{n}$ is a loop based at $H \widetilde{x}_{0}$. 
Define $g_{2}(t)=L_{n}\left(\frac{t-a}{b-a}\right)$ if $(a, b)$ is a component of $X \backslash C$ with $b-a=1 / 3^{n}$ and $t \in[a, b]$, and set $g_{2}(t)=H \widetilde{x}_{0}$ for $t \in C$.

Notice that $g_{2}$ is a loop, while $g_{1}$ is not. On one hand, $g_{2}(1)=H \widetilde{x}_{0}$ by definition. On the other hand, $[f] \in \pi_{1}\left(X, x_{0}\right) \backslash H$ so that $g_{1}(1)=H\left[f \circ \tau_{1}\right]=$ $H[f] \neq H \widetilde{x}_{0}$. We conclude that $p \circ g_{1}=p \circ g_{2}$ with $g_{1}(0)=H \widetilde{x}_{0}=g_{2}(0)$ but $g_{1}(1) \neq g_{2}(1)$. In fact, $g_{1}(t) \neq g_{2}(t)$ for all $t>0$. Figure 2 shows a schematic sketch of $g_{1}$ (upper row) and $g_{2}$. A more detailed analysis of this phenomenon can be found in [3].

The following relative version of being homotopically Hausdorff will turn out to be necessary for $p: \widetilde{X} / H \rightarrow X$ to have the unique path lifting property.

Definition. Let $H \leq \pi_{1}\left(X, x_{0}\right)$. We will call $X$ homotopically Hausdorff relative to $H$ if for every $x \in X$, for every $[\alpha] \in \widetilde{X}$ with $\alpha(1)=x$, and for every $g \in \pi_{1}\left(X, x_{0}\right) \backslash H$ there is an open subset $U \subseteq X$ with $x \in U$ such that there is no loop $\delta:\left(S^{1}, *\right) \rightarrow(U, x)$ with $[\alpha \cdot \delta \cdot \bar{\alpha}] \in H g$.

Note that $X$ is homotopically Hausdorff if and only if $X$ is homotopically Hausdorff relative to $H=\{1\}$. The significance of this relative notion is the same:

Proposition 6.3. Suppose $X$ is Hausdorff and $H \leq \pi_{1}\left(X, x_{0}\right)$. Then $\widetilde{X} / H$ is Hausdorff if and only if $X$ is homotopically Hausdorff relative to $H$.

Proof. The proof is a generalization of the proof of Lemma 2.10: suppose $X$ is homotopically Hausdorff relative to $H$. Let $H[\alpha], H[\beta] \in \widetilde{X} / H$ with $H[\alpha] \neq H[\beta]$. As in the proof of Lemma 2.10, we may assume without loss of generality that $\alpha(1)=\beta(1)$. Then $[\beta \cdot \bar{\alpha}] \in \pi_{1}\left(X, x_{0}\right) \backslash H$. Hence, there is an open subset $U \subseteq X$ with $\alpha(1) \in U$ such that there is no loop $\delta:\left(S^{1}, *\right) \rightarrow$ $(U, \alpha(1))$ with $[\alpha \cdot \delta \cdot \bar{\alpha}] \in H[\beta \cdot \bar{\alpha}]$. This implies that $H[\beta] \notin H B([\alpha], U)$ so that $H B([\beta], U) \cap H B([\alpha], U)=\emptyset$. We conclude that $\widetilde{X} / H$ is Hausdorff.

Conversely, if $X$ is not homotopically Hausdorff relative to $H$, then the proof of Proposition 6.4 below shows how to find distinct elements $H \widetilde{x}_{1}$ and $H \widetilde{x}_{2}$ of $\widetilde{X} / H$ which do not have disjoint neighborhoods.

Proposition 6.4. Let $H \leq \pi_{1}\left(X, x_{0}\right)$. If $p: \widetilde{X} / H \rightarrow X$ has the unique path lifting property, then $X$ is homotopically Hausdorff relative to $H$.

Proof. The proof is a generalization of the proof of Lemma 2.11: suppose, to the contrary, that there are $x \in X,[\alpha] \in \widetilde{X}$ with $\alpha(1)=x$, and $[\beta] \in$ $\pi_{1}\left(X, x_{0}\right) \backslash H$ such that for every open subset $U \subseteq X$ with $x \in U$ there is a $\operatorname{loop} \delta:\left(S^{1}, *\right) \rightarrow(U, x)$ with $[\alpha \cdot \delta \cdot \bar{\alpha}] \in H[\beta]$. Then $p(H[\alpha])=p(H[\beta \cdot \alpha])=x$ and $H[\alpha] \neq H[\beta \cdot \alpha]$. Let $U$ be an open subset of $X$ with $x \in U$. Choose a loop $\delta:\left(S^{1}, *\right) \rightarrow(U, x)$ with $[\alpha \cdot \delta \cdot \bar{\alpha}]=[\gamma \cdot \beta]$ for some $[\gamma] \in H$. Then $H[\alpha]=H[\alpha \cdot \delta \cdot \bar{\delta}]=H[\gamma \cdot \beta \cdot \alpha \cdot \bar{\delta}]=H[\beta \cdot \alpha \cdot \bar{\delta}] \in H B([\beta \cdot \alpha], U)$ 
and $H[\beta \cdot \alpha]=H[\gamma \cdot \beta \cdot \alpha]=H[\alpha \cdot \delta] \in H B([\alpha], U)$. Hence, any function $g:[0,1] \rightarrow \widetilde{X} / H$ with $g(t) \in\{H[\alpha], H[\beta \cdot \alpha]\}$ will be continuous and such that $p \circ g(t)=x$ for all $t \in[0,1]$. This contradicts the unique path lifting property of $p: \widetilde{X} / H \rightarrow X$.

REMARK. We note, without proof, that Lemma 2.12 generalizes similarly. The other items compiled in Proposition 2.14 have their relative analogues as well. We state them in Proposition 6.9 below and (implicitly) in our final Theorem 6.10 .

What follows are relative versions of the results of Section 4 .

Normal subgroups of countable index

Proposition 6.5. Let $H \unlhd \pi_{1}\left(X, x_{0}\right)$ be a normal subgroup. If $X$ is homotopically Hausdorff relative to $H$ and if the group $\pi\left(X, x_{0}\right) / H$ is countable, then $p: \widetilde{X} / H \rightarrow X$ has the unique path lifting property.

Proof. Let $g_{1}, g_{2}:[0,1] \rightarrow \widetilde{X} / H$ be two continuous functions with $p \circ g_{1}=$ $p \circ g_{2}$. Say, $g_{1}(t)=H\left[\alpha_{t}\right]$ and $g_{2}(t)=H\left[\beta_{t}\right]$. As in Proposition 4.3, it suffices to show that the function $f:[0,1] \rightarrow \pi_{1}\left(X, x_{0}\right) / H$ given by $f(t)=H\left[\alpha_{t} \cdot \bar{\beta}_{t}\right]$ is continuous provided $\pi_{1}\left(X, x_{0}\right) / H$ is given the finite complement topology. The proof is very similar: let $s \in[0,1]$ and $\left\{k_{1}, \ldots, k_{n}\right\} \subseteq \pi_{1}\left(X, x_{0}\right)$ be given with $f(s)=H\left[\alpha_{s} \cdot \bar{\beta}_{s}\right] \notin\left\{H k_{1}, \ldots, H k_{n}\right\}$. Since $X$ is homotopically Hausdorff relative to $H$, there is an open subset $U \subseteq X$ with $\alpha_{s}(1) \in U$ such that there is no loop $\lambda$ in $U$ with $\left[\alpha_{s} \cdot \lambda \cdot \bar{\alpha}_{s}\right] \in H k_{i}\left[\beta_{s} \cdot \bar{\alpha}_{s}\right]$ and $i \in\{1, \ldots, n\}$. By continuity of $g_{1}$ and $g_{2}$, we may choose a $\delta>0$ such that $H\left[\alpha_{t}\right] \in H B\left(\left[\alpha_{s}\right], U\right)$ and $H\left[\beta_{t}\right] \in H B\left(\left[\beta_{s}\right], U\right)$ for all $t \in[0,1]$ with $|s-t|<\delta$. Now let $t \in[0,1]$ with $|s-t|<\delta$. Then $\left[\alpha_{t}\right]=\left[\gamma \cdot \alpha_{s} \cdot \tau\right]$ and $\left[\bar{\beta}_{t}\right]=\left[\bar{\sigma} \cdot \bar{\beta}_{s} \cdot \bar{\eta}\right]$ for some $[\gamma],[\eta] \in H$ and $\tau, \sigma:[0,1] \rightarrow U$. Then $\tau \cdot \bar{\sigma}$ is a loop in $U$ based at $\alpha_{s}(1)$. Hence, $\left[\alpha_{s} \cdot(\tau \cdot \bar{\sigma}) \cdot \bar{\alpha}_{s}\right] \notin H k_{i}\left[\beta_{s} \cdot \bar{\alpha}_{s}\right]$ for all $i \in\{1, \ldots, n\}$. Since $[\gamma],[\eta] \in H$, and since $H$ is a normal subgroup of $\pi_{1}\left(X, x_{0}\right)$, it follows that $f(t)=H\left[\alpha_{t} \cdot \bar{\beta}_{t}\right]=H[\gamma]\left[\alpha_{s} \cdot(\tau \cdot \bar{\sigma}) \cdot \bar{\alpha}_{s}\right]\left[\alpha_{s} \cdot \bar{\beta}_{s}\right][\bar{\eta}] \notin\left\{H k_{1}, \ldots, H k_{n}\right\}$.

Proposition 6.6. Let $H \unlhd \pi_{1}\left(X, x_{0}\right)$ be a normal subgroup. If $X$ is first countable, locally path-connected and homotopically Hausdorff relative to $H$, and if the group $\pi_{1}\left(X, x_{0}\right) / H$ is countable, then $p: \widetilde{X} / H \rightarrow X$ is the (classical) regular covering with $p_{\#}\left(\pi_{1}(\tilde{X} / H)\right)=H$.

Proof. In view of Theorem 6.1, it suffices to show that there is an open cover $\mathcal{U}$ of $X$ such that $\pi\left(\mathcal{U}, x_{0}\right) \leq H$. Indeed, it suffices to show that for every $x \in X$ there is an open path-connected subset $U \subseteq X$ such that $x \in U$ and $[\alpha \cdot \tau \cdot \bar{\alpha}] \in H$ for every $[\alpha] \in \widetilde{X}$ and every loop $\tau:[0,1] \rightarrow U$ with $\alpha(1)=\tau(0)=\tau(1)=x$ (because then $[\alpha \cdot \tau \cdot \bar{\alpha}] \in H$ for every $[\alpha] \in \widetilde{X}$ and every loop $\tau:[0,1] \rightarrow U$ with $\alpha(1)=\tau(0)=\tau(1)$, regardless of where in $U$ the path $\alpha$ terminates). Suppose, to the contrary, that there is an $x \in X$ 
for which this is not so. Let $V_{1} \supseteq V_{2} \supseteq \cdots$ be a basis for the topology of $X$ at $x$. Fix any path-connected open subset $U_{1} \subseteq V_{1}$ with $x \in U_{1}$. Then there is an $\left[\alpha_{1}\right] \in \tilde{X}$ and a loop $\tau_{1}:[0,1] \rightarrow U_{1}$ with $\alpha_{1}(1)=\tau_{1}(0)=\tau_{1}(1)=x$ such that $\left[\alpha_{1} \cdot \tau_{1} \cdot \bar{\alpha}_{1}\right] \notin H$. Put $g_{1}=\left[\alpha_{1} \cdot \tau_{1} \cdot \bar{\alpha}_{1}\right]$. Since $X$ is homotopically Hausdorff relative to $H$ and since $g_{1} \in \pi_{1}\left(X, x_{0}\right) \backslash H$, we can find a pathconnected open subset $U_{2} \subseteq V_{2}$ with $x \in U_{2} \subseteq U_{1}$ such that there is no loop $\delta$ in $U_{2}$ with $\left[\alpha_{1} \cdot \delta \cdot \bar{\alpha}_{1}\right] \in H g_{1}$. That is, there is no loop $\delta$ in $U_{2}$ with $\left[\alpha_{1} \cdot \tau_{1} \cdot \bar{\delta} \cdot \bar{\alpha}_{1}\right] \in H$. Equivalently, there is no loop $\delta$ in $U_{2}$ with $\left[\alpha_{1} \cdot \tau_{1} \cdot \delta \cdot \bar{\alpha}_{1}\right] \in H$. Inductively, we find a basis $U_{1} \supseteq U_{2} \supseteq \cdots$ for the topology of $X$ at $x$ of path-connected open sets $U_{i}$ along with $\left[\alpha_{i}\right] \in \widetilde{X}$ and loops $\tau_{i}:[0,1] \rightarrow U_{i}$, with $\alpha_{i}(1)=\tau_{i}(0)=\tau_{i}(1)=x$, such that there is no loop $\delta$ in $U_{i+1}$ with $\left[\alpha_{i} \cdot \tau_{i} \cdot \delta \cdot \bar{\alpha}_{i}\right] \in H$. Since $H$ is a normal subgroup of $\pi_{1}\left(X, x_{0}\right)$ we see that $\left[\alpha_{1} \cdot \tau_{i} \cdot \delta \cdot \bar{\alpha}_{1}\right]=\left[\alpha_{1} \cdot \bar{\alpha}_{i}\right]\left[\alpha_{i} \cdot \tau_{i} \cdot \delta \cdot \bar{\alpha}_{i}\right]\left[\alpha_{1} \cdot \bar{\alpha}_{i}\right]^{-1} \notin H$ for any loop $\delta$ in $U_{i+1}$.

As in the proof of Proposition 4.6, for each increasing sequence $i_{1}<i_{2}$ $<\cdots$ of natural numbers we can now define a continuous loop $\tau:[0,1] \rightarrow X$ by the formula $\tau(t)=\tau_{i_{k}}\left(2-2^{k}(1-t)\right)$ when $1-1 / 2^{k-1} \leq t \leq 1-1 / 2^{k}$ and $\tau(1)=x$. Then distinct sequences $i_{1}<i_{2}<\cdots$ and $i_{1}^{\prime}<i_{2}^{\prime}<\cdots$ yield distinct cosets $H\left[\alpha_{1} \cdot \tau \cdot \bar{\alpha}_{1}\right]$ and $H\left[\alpha_{1} \cdot \tau^{\prime} \cdot \bar{\alpha}_{1}\right]$ of $\pi_{1}\left(X, x_{0}\right) / H$, respectively. To see why, assume, without loss of generality, that $i_{1}<i_{1}^{\prime}$. If we had $H\left[\alpha_{1} \cdot \tau^{\prime} \cdot \bar{\alpha}_{1}\right]=H\left[\alpha_{1} \cdot \tau \cdot \bar{\alpha}_{1}\right]$, then $\left[\alpha_{1} \cdot \tau \cdot \bar{\tau}^{\prime} \cdot \bar{\alpha}_{1}\right] \in H$. This would imply that there is a loop $\delta$ in $U_{i_{1}+1}$ such that $\left[\alpha_{1} \cdot \tau_{i_{1}} \cdot \delta \cdot \bar{\alpha}_{1}\right] \in H$, which was ruled out.

Since $\pi_{1}\left(X, x_{0}\right) / H$ was assumed to be countable, we have arrived at our desired contradiction.

Special normal subgroups of arbitrary index

Proposition 6.7. Let $\mathcal{C}$ be some collection of open covers of $X$ and $H=$ $\bigcap_{\mathcal{U} \in \mathcal{C}} \pi\left(\mathcal{U}, x_{0}\right)$. Then $p: \widetilde{X} / H \rightarrow X$ has the unique path lifting property.

Proof. We will show that for any two continuous maps $g_{1}:[0,1] \rightarrow$ $\widetilde{X} / H$ and $g_{2}:[0,1] \rightarrow \widetilde{X} / H$ such that $p \circ g_{1}=p \circ g_{2}$, the set $\{t \in$ $\left.[0,1] \mid g_{1}(t)=g_{2}(t)\right\}$ is either empty or all of $[0,1]$. For every $t \in[0,1]$ choose continuous maps $\alpha_{t}, \beta_{t}:[0,1] \rightarrow X$ with $g_{1}(t)=H\left[\alpha_{t}\right]$ and $g_{2}(t)=$ $H\left[\beta_{t}\right]$. Suppose, to the contrary, that there are $r, s \in[0,1]$ with $H\left[\alpha_{r}\right] \neq$ $H\left[\beta_{r}\right]$ and $H\left[\alpha_{s}\right]=H\left[\beta_{s}\right]$. Moreover, assume, without loss of generality, that $r<s$.

Since $\left[\alpha_{r} \cdot \bar{\beta}_{r}\right] \notin H$, then by assumption, there is an open cover $\mathcal{U} \in \mathcal{C}$ of $X$ such that $\left[\alpha_{r} \cdot \bar{\beta}_{r}\right] \notin \pi\left(\mathcal{U}, x_{0}\right)$. Let $v$ be the greatest lower bound for the set $A=\left\{t \in[r, s] \mid\left[\alpha_{t} \cdot \bar{\beta}_{t}\right] \in \pi\left(\mathcal{U}, x_{0}\right)\right\}$. Let $x=\alpha_{v}(1)=\beta_{v}(1)$. Choose $U \in \mathcal{U}$ with $x \in U$. By continuity of $g_{1}$ and $g_{2}$, there is a $\delta>0$ such that $H\left[\alpha_{t}\right] \in H B\left(\left[\alpha_{v}\right], U\right)$ and $H\left[\beta_{t}\right] \in H B\left(\left[\beta_{v}\right], U\right)$ for all $t \in[0,1]$ with $|t-v|<\delta$. 
(i) Suppose $\left[\alpha_{v} \cdot \bar{\beta}_{v}\right] \in \pi\left(\mathcal{U}, x_{0}\right)$. Then $r<v \leq s$. Choose $t \in(r, v)$ such that $|t-v|<\delta$. By definition of $B\left(\left[\alpha_{v}\right], U\right)$ and $B\left(\left[\beta_{v}\right], U\right)$, there are continuous maps $\tau_{1}, \tau_{2}:[0,1] \rightarrow U$ such that $H\left[\alpha_{t}\right]=H\left[\alpha_{v} \cdot \tau_{1}\right]$ and $H\left[\beta_{t}\right]=H\left[\beta_{v} \cdot \tau_{2}\right]$. So, $\left[\alpha_{t}\right]=\left[\delta_{1} \cdot \alpha_{v} \cdot \tau_{1}\right]$ and $\left[\bar{\beta}_{t}\right]=\left[\bar{\tau}_{2} \cdot \bar{\beta}_{v} \cdot \bar{\delta}_{2}\right]$ for some $\left[\delta_{i}\right] \in H \leq \pi\left(\mathcal{U}, x_{0}\right)$.

Since $\tau_{1} \cdot \bar{\tau}_{2}$ is a loop in $U$, we have $\left[\alpha_{v} \cdot\left(\tau_{1} \cdot \bar{\tau}_{2}\right) \cdot \bar{\alpha}_{v}\right] \in \pi\left(\mathcal{U}, x_{0}\right)$. Consequently, $\left[\alpha_{t} \cdot \bar{\beta}_{t}\right]=\left[\delta_{1}\right]\left[\alpha_{v} \cdot\left(\tau_{1} \cdot \bar{\tau}_{2}\right) \cdot \bar{\alpha}_{v}\right]\left[\alpha_{v} \cdot \bar{\beta}_{v}\right]\left[\bar{\delta}_{2}\right] \in \pi\left(\mathcal{U}, x_{0}\right)$. However, $t<v$, so that $v$ is not a lower bound for the set $A$; a contradiction.

(ii) Now suppose $\left[\alpha_{v} \cdot \bar{\beta}_{v}\right] \notin \pi\left(\mathcal{U}, x_{0}\right)$. Then $r \leq v<s$. Using an argument similar to part (i), we see that $\left[\alpha_{t} \cdot \bar{\beta}_{t}\right] \notin \pi\left(\mathcal{U}, x_{0}\right)$ for all $t \in[v, s)$ with $|t-v|<\delta$. Choose $u \in(v, s)$ with $|u-v|<\delta$. Then $u$ is a lower bound for the set $A$, which is greater than $v$; a contradiction.

Proposition 6.8. Let $K$ be the kernel of $\varphi: \pi_{1}\left(X, x_{0}\right) \rightarrow \check{\pi}_{1}\left(X, x_{0}\right)$. Then $p: \widetilde{X} / K \rightarrow X$ has the unique path lifting property.

Proof. The proof is very similar to that of Proposition 6.7 and we use the same setup of the first paragraph upon replacing $H$ by $K$.

However, this time, $\left[\alpha_{r} \cdot \bar{\beta}_{r}\right] \notin K$ implies that there is a normal cover $\mathcal{U}$ of $X$ such that $p_{\mathcal{U} \#}\left(\left[\alpha_{r} \cdot \bar{\beta}_{r}\right]\right) \neq 1 \in \pi_{1}(N(\mathcal{U}), *)$, and we let $v$ be the greatest lower bound of the set $A=\left\{t \in[r, s] \mid p_{\mathcal{U} \#}\left(\left[\alpha_{t} \cdot \bar{\beta}_{t}\right]\right)\right]=$ $\left.1 \in \pi_{1}(N(\mathcal{U}), *)\right\}$. Again, we put $x=\alpha_{v}(1)=\beta_{v}(1)$. Since the collection $\left\{p_{\mathcal{U}}^{-1}(\operatorname{St}(U, N(\mathcal{U}))) \mid U \in \mathcal{U}\right\}$ is an open cover of $X$, we may choose $U \in \mathcal{U}$ so that $x \in V=p_{\mathcal{U}}^{-1}(\operatorname{St}(U, N(\mathcal{U})))$. Accordingly, we choose $\delta>0$ such that $K\left[\alpha_{t}\right] \in K B\left(\left[\alpha_{v}\right], V\right)$ and $K\left[\beta_{t}\right] \in K B\left(\left[\beta_{v}\right], V\right)$ for all $t \in[0,1]$ with $|t-v|<\delta$.

(i) Suppose $p_{\mathcal{U} \#}\left(\left[\alpha_{v} \cdot \bar{\beta}_{v}\right]\right)=1 \in \pi_{1}(N(\mathcal{U}), *)$. Then $r<v \leq s$. Choose $t \in(r, v)$ such that $|t-v|<\delta$. As before, there are paths $\tau_{1}, \tau_{2}:[0,1] \rightarrow V$ such that $\left[\alpha_{t}\right]=\left[\delta_{1} \cdot \alpha_{v} \cdot \tau_{1}\right]$ and $\left[\bar{\beta}_{t}\right]=\left[\bar{\tau}_{2} \cdot \bar{\beta}_{v} \cdot \bar{\delta}_{2}\right]$ for some $\left[\delta_{i}\right] \in K$. Since $\tau_{1} \cdot \bar{\tau}_{2}$ is a loop in $V$, the loop $\left(p_{\mathcal{U}} \circ \tau_{1}\right) \cdot\left(\overline{\mathcal{M}_{\mathcal{U}} \circ \tau_{2}}\right)$ lies in the open star of the vertex corresponding to $U$ in $N(\mathcal{U})$, where it can be homotoped to that vertex. Also, because both $\left[\delta_{1}\right]$ and $\left[\delta_{2}\right]$ lie in the kernel of $\pi_{1}\left(X, x_{0}\right) \rightarrow \check{\pi}_{1}\left(X, x_{0}\right)$, we have $p_{\mathcal{U} \#}\left(\left[\delta_{1}\right]\right)=p_{\mathcal{U} \#}\left(\left[\bar{\delta}_{2}\right]\right)=1 \in \pi_{1}(N(\mathcal{U}), *)$. Consequently,

$$
\begin{aligned}
p_{\mathcal{U} \#}\left(\left[\alpha_{t} \cdot \bar{\beta}_{t}\right]\right) & =p_{\mathcal{U} \#}\left(\left[\delta_{1} \cdot \alpha_{v} \cdot \tau_{1} \cdot \bar{\tau}_{2} \cdot \bar{\beta}_{v} \cdot \bar{\delta}_{2}\right]\right) \\
& =p_{\mathcal{U} \#}\left(\left[\alpha_{v} \cdot \bar{\beta}_{v}\right]\right)=1 \in \pi_{1}(N(\mathcal{U}), *) .
\end{aligned}
$$

However, $t<v$, so that $v$ is not a lower bound for the set $A$ and we have the desired contradiction.

(ii) As was the case in the proof of Proposition 6.7, a similar contradiction occurs when $p_{\mathcal{U} \#}\left(\left[\alpha_{v} \cdot \bar{\beta}_{v}\right]\right) \neq 1 \in \pi_{1}(N(\mathcal{U}), *)$.

The following proposition (whose proof can be found in [3]) shows that, as before, all relevant properties depend only on unique path lifting. 
Proposition 6.9. Let $H \leq \pi_{1}\left(X, x_{0}\right)$ be a subgroup such that $p: \widetilde{X} / H$ $\rightarrow X$ has the unique path lifting property. Then

(a) $\tilde{X} / H$ is path-connected and locally path-connected.

(b) $p_{\#}: \pi_{1}\left(\widetilde{X} / H, H \widetilde{x}_{0}\right) \rightarrow \pi_{1}\left(X, x_{0}\right)$ is a monomorphism onto $H$.

(c) For every path-connected and locally path-connected space $Y$, for every continuous $f:(Y, y) \rightarrow(X, x)$ and every $H \widetilde{x} \in \widetilde{X} / H$ with $p(H \widetilde{x})=x$, there is a unique continuous $g:(Y, y) \rightarrow(\widetilde{X} / H, H \widetilde{x})$ with $p \circ g=f$, provided $f_{\#}\left(\pi_{1}(Y, y)\right) \leq p_{\#}\left(\pi_{1}(\widetilde{X} / H, H \widetilde{x})\right)$.

We sketch the short proof for completeness.

Proof. (a) Note that the quotient map $q: \widetilde{X} \rightarrow \widetilde{X} / H$ is open.

(b) Injectivity of $p_{\#}$ follows from Proposition 2.4 and the unique path lifting property of $p: \widetilde{X} / H \rightarrow X$. Now, let $[\alpha] \in H$ be given and let $\widetilde{\alpha}:([0,1], 0) \rightarrow\left(\widetilde{X}, \widetilde{x}_{0}\right)$ be the standard lift of $\alpha:([0,1], 0) \rightarrow\left(X, x_{0}\right)$. Then $[q \circ \widetilde{\alpha}] \in \pi_{1}\left(\widetilde{X} / H, H \widetilde{x}_{0}\right)$, because $q \circ \widetilde{\alpha}(0)=H \widetilde{x}_{0}=H[\alpha]=q \circ \widetilde{\alpha}(1)$, and $[\alpha]=p_{\#}([q \circ \widetilde{\alpha}])$. Conversely, let $[\bar{\gamma}] \in \pi_{1}\left(\widetilde{X} / H, H \widetilde{x}_{0}\right)$ be given and let $\widetilde{\gamma}:([0,1], 0) \rightarrow\left(\widetilde{X}, \widetilde{x}_{0}\right)$ be the standard lift of $\gamma=p \circ \bar{\gamma}:([0,1], 0) \rightarrow\left(X, x_{0}\right)$. Then $\bar{\gamma}=q \circ \widetilde{\gamma}$, since $p: \widetilde{X} / H \rightarrow X$ has the unique path lifting property. Hence, $H \widetilde{x}_{0}=\bar{\gamma}(1)=H \widetilde{\gamma}(1)=H[\gamma]$, so that $p_{\#}([\bar{\gamma}])=[\gamma] \in H$.

(c) As in Proposition 2.4, define $g(w)=H[\alpha \cdot(f \circ \tau)]$, where $\widetilde{x}=[\alpha] \in \widetilde{X}$ and $\tau:[0,1] \rightarrow Y$ is any path from $\tau(0)=y$ to $\tau(1)=w$. Then $g$ is welldefined, because we have $[\alpha] f_{\#}\left(\pi_{1}(Y, y)\right)[\bar{\alpha}] \subseteq[\alpha] p_{\#}\left(\pi_{1}(\widetilde{X} / H, H[\alpha])\right)[\bar{\alpha}]=$ $[p \circ q \circ \widetilde{\alpha}] p_{\#}\left(\pi_{1}(\widetilde{X} / H, H[\alpha])\right)[p \circ q \circ \widetilde{\bar{\alpha}}]=p_{\#}\left(\pi_{1}\left(\widetilde{X} / H, H \widetilde{x}_{0}\right)\right)=H$, by part (b). All remaining properties of $g$ are verified as before.

In order to summarize the results of this section, we make the following definition.

Definition. Let $H \unlhd \pi_{1}\left(X, x_{0}\right)$ be a normal subgroup. We call a map $\bar{p}: \bar{X} \rightarrow X$ a generalized regular covering of $X$ with respect to $H$ if the following three properties are satisfied, which uniquely characterize $\bar{p}: \bar{X}$ $\rightarrow X$ (as in Section 1.1):

$\left(\mathrm{R}_{1}\right) \bar{X}$ is path-connected and locally path-connected.

$\left(\mathrm{R}_{2}\right) \bar{p}: \bar{X} \rightarrow X$ is a continuous surjection and $\bar{p}_{\#}: \pi_{1}(\bar{X}) \rightarrow \pi_{1}(X)$ is a monomorphism onto $H$.

$\left(\mathrm{R}_{3}\right)$ For every path-connected and locally path-connected space $Y$, for every continuous $f:(Y, y) \rightarrow(X, x)$ and every $\bar{x} \in \bar{X}$ with $\bar{p}(\bar{x})=x$, there is a unique continuous $g:(Y, y) \rightarrow(\bar{X}, \bar{x})$ with $\bar{p} \circ g=f$, provided $f_{\#}\left(\pi_{1}(Y, y)\right) \leq \bar{p}_{\#}\left(\pi_{1}(\bar{X}, \bar{x})\right)$.

Combining Propositions 6.7, 6.8 and 6.5 with Proposition 6.9, we obtain the following generalization of Theorems 4.4 and 4.10 : 
THEOREM 6.10. Let $H \unlhd \pi_{1}\left(X, x_{0}\right)$ be a normal subgroup and suppose that one of the following holds:

(i) $H=\bigcap_{\mathcal{U} \in \mathcal{C}} \pi\left(\mathcal{U}, x_{0}\right)$ for some collection $\mathcal{C}$ of open covers of $X$, or

(ii) $H$ is the kernel of $\varphi: \pi_{1}\left(X, x_{0}\right) \rightarrow \check{\pi}_{1}\left(X, x_{0}\right)$, or

(iii) $X$ is homotopically Hausdorff relative to $H$ and $\pi_{1}\left(X, x_{0}\right) / H$ is countable.

Then

(a) The map $p: \widetilde{X} / H \rightarrow X$ is a generalized regular covering with respect to $H$.

(b) The group $G=\operatorname{Aut}(\widetilde{X} / H \stackrel{p}{\rightarrow} X)$ of covering transformations is isomorphic to $\pi_{1}\left(X, x_{0}\right) / H$ and it acts freely and transitively on every fiber $p^{-1}(\{x\})$ with $x \in X$.

(c) If $X$ is locally path-connected, then $p: \widetilde{X} / H \rightarrow X$ is an open map.

(d) If $X$ is Hausdorff or metrizable, then so is $\widetilde{X} / H$.

(e) If $X$ is metrizable, then there is a metric for $\widetilde{X} / H$ such that $G$ acts by isometry.

\section{References}

[1] R. A. Alo and H. L. Shapiro, Normal Topological Spaces, Cambridge Univ. Press, 1974.

[2] D. K. Biss, The topological fundamental group and generalized covering spaces, Topology Appl. 124 (2002), 355-371.

[3] W. A. Bogley and A. J. Sieradski, Universal path spaces, preprint, Oregon State Univ. and Univ. of Oregon, 1998.

[4] J. W. Cannon, Geometric group theory, in: R. J. Daverman and R. B. Sher (eds.), Handbook of Geometric Topology, Elsevier, 2002, 261-305.

[5] J. W. Cannon and G. R. Conner, On the fundamental groups of one-dimensional spaces, Topology Appl. 153 (2006), 2648-2672.

[6] G. R. Conner and J. W. Lamoreaux, On the existence of universal covering spaces for metric spaces and subsets of the Euclidean plane, Fund. Math. 187 (2005), 95-110.

[7] G. R. Conner, M. Meilstrup, D. Repovš, A. Zastrow, and M. Željko, On small nulhomotopies of loops, preprint, 2007.

[8] M. L. Curtis and M. K. Fort, Jr., Homotopy groups of one-dimensional spaces, Proc. Amer. Math. Soc. 8 (1957), 577-579.

[9] K. Eda and K. Kawamura, The fundamental groups of one-dimensional spaces, Topology Appl. 87 (1998), 163-172.

[10] P. Fabel, A monomorphism theorem for the inverse limit of nested retracts, preprint, 2005; http://front.math.ucdavis.edu/math.AT.

[11] H. Fischer and C. R. Guilbault, On the fundamental groups of trees of manifolds, Pacific J. Math. 221 (2005), 49-79.

[12] H. Fischer and A. Zastrow, The fundamental groups of subsets of closed surfaces inject into their first shape groups, Algebr. Geom. Topol. 5 (2005), 1655-1676.

[13] R. H. Fox, On shape, Fund. Math. 74 (1972), 47-71. 
[14] R. H. Fox, Shape theory and covering spaces, in: Lecture Notes in Math. 375, Springer, 1974, 71-90.

[15] L. J. Hernández-Paricio, Fundamental pro-groupoids and covering projections, Fund. Math. 156 (1998), 1-31.

[16] S.. Koçak, On the fundamental theorem of overlays, Note Mat. 10 (1990), 355-362.

[17] G. Kozlowski and J. Segal, Local behavior and the Vietoris and Whitehead theorems in shape theory, Fund. Math. 99 (1978), 213-225.

[18] K. Kuperberg, Two Vietoris-type isomorphism theorems in Borsuk's theory of shape, concerning the Vietoris-Čech homology and Borsuk's fundamental groups, in: Studies in Topology (Charlotte, NC, 1974), Academic Press, 1975, 285-313.

[19] S. Lubkin, Theory of covering spaces, Trans. Amer. Math. Soc. 104 (1962), 205-238.

[20] S. Mardešić and V. Matijević, Classifying overlay structures of topological spaces, Topology Appl. 113 (2001), 167-209.

[21] S. Mardešić and J. Segal, Shape Theory: the Inverse System Approach, NorthHolland Math. Library 26, North-Holland, 1982.

[22] W. S. Massey, Algebraic Topology: an Introduction, Grad. Texts in Math. 56, Springer, 1977.

[23] V. Matijević, Classifying finite-sheeted covering mappings of paracompact spaces, Rev. Mat. Complut. 16 (2003), 311-327.

[24] J. Mayer and L. Oversteegen, A topological characterization of $\mathbb{R}$-trees, Trans. Amer. Math. Soc. 320 (1990), 395-415.

[25] E. H. Spanier, Algebraic Topology, McGraw-Hill, 1966.

[26] A. Zastrow, The non-abelian Specker-group is free, J. Algebra 229 (2000), 55-85.

[27] - Construction of an infinitely generated group that is not a free product of surface groups and abelian groups, but which acts freely on an $\mathbb{R}$-tree, Proc. Roy. Soc. Edinburgh Sect. A 128 (1998), 433-445.

[28] - Generalized $\pi_{1}$-determined covering spaces, unpublished notes, 1996; http://math.univ.gda.pl/_zastrow/gcv_run.dvi.

Department of Mathematical Sciences

Ball State University

Muncie, IN 47306, U.S.A.

E-mail: fischer@math.bsu.edu
Institute of Mathematics University of Gdańsk Wita Stwosza 57 80-952 Gdańsk, Poland E-mail: zastrow@math.univ.gda.pl

Received 23 November 2005; in revised form 25 October 2007 